\title{
Completion of Ontologies and Ontology Networks
}

\author{
by
}

\section{Zlatan Dragisic}

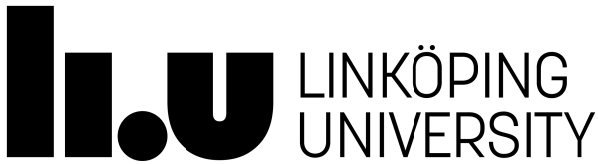

Department of Computer and Information Science Linköping University

SE-581 83 Linköping, Sweden 
Copyright (C) 2017 Zlatan Dragisic

ISBN 978-91-7685-522-5

ISSN 0345-7524

Printed by LiU Tryck 2017

URL: http://urn.kb.se/resolve?urn=urn:nbn:se:liu:diva-139487 


\section{Abstract}

The World Wide Web contains large amounts of data, and in most cases this data has no explicit structure. The lack of structure makes it difficult for automated agents to understand and use such data. A step towards a more structured World Wide Web is the Semantic Web, which aims at introducing semantics to data on the World Wide Web. One of the key technologies in this endeavour are ontologies, which provide a means for modeling a domain of interest and are used for search and integration of data.

In recent years many ontologies have been developed. To be able to use multiple ontologies it is necessary to align them, i.e., find inter-ontology relationships. However, developing and aligning ontologies is not an easy task and it is often the case that ontologies and their alignments are incorrect and incomplete. This can be a problem for semantically-enabled applications. Incorrect and incomplete ontologies and alignments directly influence the quality of the results of such applications, as wrong results can be returned and correct results can be missed. This thesis focuses on the problem of completing ontologies and ontology networks.

The contributions of the thesis are threefold. First, we address the issue of completing the is-a structure and alignment in ontologies and ontology networks. We have formalized the problem of completing the is-a structure in ontologies as an abductive reasoning problem and developed algorithms as well as systems for dealing with the problem. With respect to the completion of alignments, we have studied system performance in the Ontology Alignment Evaluation Initiative, a yearly evaluation campaign for ontology alignment systems. We have also addressed the scalability of ontology matching, which is one of the current challenges, by developing an approach for reducing the search space when generating the alignment.

Second, high quality completion requires user involvement. As users' time and effort are a limited resource we address the issue of limiting and facilitating user interaction in the completion process. We have conducted a broad study of state-of-the-art ontology alignment systems and identified different issues related to the process. We have also conducted experiments to assess the impact of user errors in the completion process.

While the completion of ontologies and ontology networks can be done at any point in the life-cycle of ontologies and ontology networks, some of the issues can be addressed already in the development phase. The third contribution of the thesis addresses this by introducing ontology completion and ontology alignment into an existing ontology development methodology.

The work is funded by the Swedish Research Council (2010-4759), the Swedish National Graduate School in Computer Science (CUGS), the Swedish e-Science Research Centre (SeRC) and the EU FP'7 project VALCRI (FP'IP-608142). 



\section{Populärvetenskaplig sammanfattning}

Föreställ dig att vi är intresserade av att ta reda på hur många Oscarsnomineringar och priser som varje skådespelare i någon film har haft. Om vi försöker ta reda på den här informationen på Internet skulle vi troligen behöva besöka flera webbplatser eftersom ingen av de befintliga webbplatserna ger oss informationen direkt. Till exempel skulle vi först behöva besöka en webbplats som innehåller en lista med namn på alla skådespelarna i filmen. Därefter skulle vi behöva besöka en annan webbplats som har en lista över Oscarsnomineringarna genom åren. Till sist kan vi kombinera informationen från de ovanstående webbplatserna, och räkna antalet nomineringar per skådespelare.

Om denna information finns tillgänglig på webben, varför kan inte besvarandet av frågan automatiseras? På grund av den arkitektur och design som webbplatser använder idag är informationen inte direkt användbar av automatiserade agenter. Innehållet på en webbplats som kan tolkas av agenten innehåller vanligtvis bara den information som behövs för att korrekt representera webbplatsen i en webbläsare. Ett sätt att hantera detta på är att även inkludera maskinläsbar information som beskriver innehållet på webbplatsen. På så sätt kan automatiserade agenter förstå den information som en webbplats innehåller. Vidare kan de relatera och kombinera den med information från andra webbplatser kodade på liknande sätt och besvara frågan självständigt. Detta är ett av målen för den semantiska webben, som ska vara en förlängning av den nuvarande webben, så att kunskapen och informationen är läsbar och förståelig för maskinerna. En av de tekniker som används för att uppnå detta är ontologier. Ontologier gör det möjligt att ange ordförråd som används för att beskriva information på webben. De gör det möjligt att definiera termer och relationer mellan dem, vilket en automatiserad agent kan använda för att tolka innehållet på webbplatsen. Ontologier är emellertid ofta inte fullständiga vilket kan leda till ofullständiga resultat. I de fall då det är nödvändigt att kombinera information från flera webbplatser, som i vårt exempel, kan det vara så att ontologier använder olika termer för att definiera samma begrepp. Till exempel kan en webbplats använda termen skådespelare medan den andra kan använda termen artist för att beskriva begreppet skådespelare. Denna heterogenitet gör det svårt att kombinera information från flera källor. Därför är det nödvändigt att identifiera sambandet mellan termer av de ontologier som används av olika webbplatser. Uppsättningen av dessa relationer kallas en justering.

Inriktningen på denna avhandling är att komplettera ontologier och ontologinätverk, dvs. ontologier kopplade till justeringar. Högkvalitativ komplettering och justering av ontologier kräver användarens engagemang för att bestämma om ett visst förhållande håller i en ontologi eller mellan ontologier eftersom det kräver kunskap om den domän som ontologierna beskriver. Bidragen av denna avhandling är följande. Först utformade vi metoder för att komplettera den vanligaste typen av relationer i ontologier, dvs is-a- 
relationer. Is-a-relationer används för att beskriva att ett visst begrepp är en sidotyp av (mer specifik än) någon annan ( $\mathrm{t}$ ex ett träd är en växt). Därefter är det ett problem att beräkning av justeringarna kräver att man jämför alla termer $i$ en ontologi med alla termer i en annan ontologi som kan kräva mycket tid och beräkningsresurser. Därför diskuterar vi en metod som skulle begränsa antalet jämförelser till endast de som är bra kandidater. Vidare tittade vi på hur vi kan hjälpa användaren i kompletteringsprocessen så att han/hon inte är överväldigad av förslagen från systemen. Slutligen såg vi på hur vi kan integrera kompletteringen och justeringen av ontologier i utvecklingsfasen för ontologierna och därigenom garantera en högre kvalitet på ontologierna. 


\section{Acknowledgements}

The period of my $\mathrm{PhD}$ studies, while often tough and challenging, has also been fun and rewarding. It has not only produced this thesis, but has also helped me develop on a personal level. Many people contributed to this, for which I am grateful.

First and foremost my deepest gratitude goes to my supervisor, Patrick Lambrix. I thank you for believing in me, your calmness, words of support, and for being available whenever I needed advice. You are the kind of supervisor every $\mathrm{PhD}$ student can only wish for. Working with you has made me a better researcher and a better person.

To Nahid Shahmehri, my co-supervisor and the head of the division, I thank you for making ADIT a rewarding work place, all the help during these years and your genuine interest for my well-being. I thank my other co-supervisors, Marco Kuhlmann and Fang Wei-Kleiner, for interesting and useful discussions related to my research.

During my studies I have had an opportunity to collaborate with a number of researchers. A special thank you goes to Eva Blomqvist, Henrik Eriksson, Robin Keskisärkkä and Karl Hammar for welcoming me into the VALCRI project and for all the support during the last three years. The experience of working with you has definitely helped me improve on many levels.

I am grateful to my co-authors, Craig Anslow, Tania Cerquitelli, Agnese Chiatti, Daniel Faria, Ernesto Jiménez-Ruiz, and Catia Pesquita for all the discussion and help related to our papers, which in the end, contributed to this thesis.

I thank all the previous and current ADIT members for making ADIT an enjoyable work place, and at the same time for being inspirational and motivating. A big thank you goes to Marcus Bendtsen, Vengatanathan Krishnamoorthi, Jose Peña, and Dag Sonntag for interesting and fun discussions about nothing (and everything) during lunches and fikas. A special thank you to Valentina Ivanova who was many times my travel companion, and with whom I had interesting and useful discussions both related to research and life in general.

During the days when I had a lunch box with me I enjoyed the company of the Ljusgården lunch group. To all of you thank you for all the fun and unusual topics, which often made the rest of the day much easier to cope with.

I am grateful to Brittany Shahmehri and Marco Kuhlmann for thoroughly proofreading this thesis and their valuable comments on how to improve it.

I thank Karin Baardsen, Marie Johansson, Inger Norén, and Eva Pelayo Danils who helped with various administrative issues during my studies. I am especially grateful to Anne Moe who made all the administration related to the PhD studies simple and easy to follow. 
To all my friends, thank you for all the fun moments we spent during this period. Thank you for your support, enthusiasm and most importantly for being the second family away from home.

I am grateful to my family, especially my parents and my brother. Thank you for the sacrifices you have made during this time, for all the support and love and for being there whenever I needed you. Last but not least, I would like to express my deepest gratitude to my love, my wife Svjetlana. Thank you for having endless patience to listen to my ramblings and my concerns. This period had ups and downs for both of us, but regardless I could count on your love at any point. Thank you for your advices and for brightening my days.

Zlatan Dragisic August 2017

Linköping, Sweden 


\section{List of publications}

\section{Included papers}

Paper I P. Lambrix, F. Wei-Kleiner, and Z. Dragisic. Completing the isa structure in light-weight ontologies, Journal of Biomedical Semantics, volume 6, number 12, 2015.

Paper II P. Lambrix, Z. Dragisic, and V. Ivanova. Get My Pizza Right: Repairing Missing is-a Relations in $\mathcal{A L C}$ Ontologies, In Proceedings of the 2nd Joint International Semantic Technology Conference - JIST 2012, volume 7774 of Lecture Notes in Computer Science, pages 17-32, Nara, Japan, 2012. (revised)

Paper III A. Chiatti, Z. Dragisic, T. Cerquitelli, and P. Lambrix. Reducing the search space in ontology alignment using clustering techniques and topic identification, In Proceedings of the 8th International Conference on Knowledge Capture - K-CAP 2015, paper 21, Palisades, NY, USA, 2015. (revised)

Paper IV Z. Dragisic, V. Ivanova, H. Li, and P. Lambrix. Experiences from the Anatomy track in the Ontology Alignment Evaluation Initiative, submitted.

Paper V Z. Dragisic, V. Ivanova, P. Lambrix, D. Faria, E. Jiménez-Ruiz, C. Pesquita. User Validation in Ontology Alignment, In Proceedings of the 15th International Semantic Web Conference ISWC 2016, volume 9981 of Lecture Notes in Computer Science, pages 200-217, Kobe, Japan, 2016.

Paper VI Z. Dragisic, P. Lambrix, and E. Blomqvist. Integrating Ontology Debugging and Matching into the eXtreme Design Methodology, In Proceedings of the 6th Workshop on Ontology and Semantic Web Patterns - WOP 2015, volume 1461 of CEUR Workshop Proceedings, paper 1, Bethlehem, PA, USA, 2015.

\section{Other publications}

- P. Lambrix, F. Wei-Kleiner, Z. Dragisic, and V. Ivanova. Repairing Missing Is-a structure in ontologies is an abductive reasoning problem, In Proceedings of the 2nd International Workshop on Debugging Ontologies and Ontology Mappings - WoDOOM 2013, volume 999 of CEUR Workshop Proceedings, pages 33-44, Montpellier, France, 2013.

- Z. Dragisic, P. Lambrix, and F. Wei-Kleiner. Completing the isa structure of biomedical ontologies, In Proceedings of the 10th International Conference on Data Integration in the Life Sciences - 
DILS 2014, volume 8574 of Lecture Notes in Bioinformatics, pages 66-80, Lisbon, Portugal, 2014.

- F. Wei-Kleiner, Z. Dragisic, and P. Lambrix. Abduction Framework for Repairing Incomplete $\mathcal{E} \mathcal{L}$ Ontologies: Complexity Results and Algorithms, In Proceedings of the 28th AAAI Conference on Artificial Intelligence - AAAI 2014, pages 1120-1127, Quebec City, Canada, 2014.

- Z. Dragisic, P. Lambrix, and F. Wei-Kleiner. A System for Debugging Missing Is-a Structure in $\mathcal{E} \mathcal{L}$ Ontologies, In Proceedings of the 3rd International Workshop on Debugging Ontologies and Ontology Mappings - WoDOOM 2014, volume 1162 of CEUR Workshop Proceedings, pages 51-58, Anissaras/Hersonissou, Greece, 2014. Demo.

- Z. Dragisic. Completing the Is-a Structure in Description Logics Ontologies, Licentiate Thesis, Department of Computer and Information Science, Linköping University, Linköping, Sweden, 2014.

- P. Lambrix, Z. Dragisic, V. Ivanova, C. Anslow. Visualization for Ontology Evolution, In Proceedings of the 2nd International Workshop on Visualization and Interaction for Ontologies and Linked Data - VOILA 2016, volume 1704 of CEUR Workshop Proceedings, pages 54-67, Kobe, Japan, 2016.

- B. Cuenca Grau, Z. Dragisic, K. Eckert, J. Euzenat, A. Ferrara, R. Granada, V. Ivanova, E. Jiménez-Ruiz, A. O. Kempf, P. Lambrix, A. Nikolov, H. Paulheim, D. Ritze, F. Scharffe, P. Shvaiko, C. Trojahn and O. Zamazal. Results of the Ontology Alignment Evaluation Initiative 2013, In Proceedings of the 8th International Workshop on Ontology Matching - OM 2013, volume 1111 of CEUR Workshop Proceedings, pages 61-100, Sydney, Australia, 2013.

- Z. Dragisic, K. Eckert, J. Euzenat, D. Faria, A. Ferrara, R. Granada, V. Ivanova, E. Jiménez-Ruiz, A. O. Kempf, P. Lambrix, S. Montanelli, H. Paulheim, D. Ritze, P. Shvaiko, A. Solimando, C. Trojahn, O. Zamazal, and B. Cuenca Grau. Results of the Ontology Alignment Evaluation Initiative 2014, In Proceedings of the 9th International Workshop on Ontology Matching - OM 2014, volume 1317 of CEUR Workshop Proceedings, pages 61-104, Riva del Garda, Italy, 2014.

- M. Cheatham, Z. Dragisic, J. Euzenat, D. Faria, A. Ferrara, G. Flouris, I. Fundulaki, R. Granada, V. Ivanova, E. Jiménez-Ruiz, P. Lambrix, S. Montanelli, C. Pesquita, T. Saveta, P. Shvaiko, A. Solimando, C. Trojahn, and O. Zamazal. Results of the Ontology Alignment Evaluation Initiative 2015, In Proceedings of the 10th International Workshop on Ontology Matching - OM 2015, volume 1545 of CEUR Workshop Proceedings, pages 60-115, Bethlehem, PA, USA, 2015. 
- M. Achichi, M. Cheatham, Z. Dragisic, J. Euzenat, D. Faria, A. Ferrara, G. Flouris, I. Fundulaki, I. Harrow, V. Ivanova, E. Jiménez-Ruiz, E. Kuss, P. Lambrix, H. Leopold, H. Li, C. Meilicke, S. Montanelli, C. Pesquita, T. Saveta, P. Shvaiko, A. Splendiani, H. Stuckenschmidt, K. Todorov, C. Trojahn, and O. Zamazal. Results of the Ontology Alignment Evaluation Initiative 2016, In Proceedings of the 11th International Workshop on Ontology Matching - OM 2016, volume 1766 of CEUR Workshop Proceedings, pages 73-129, Kobe, Japan, 2016. 



\section{Contents}

I Summary 1

1 Introduction $\quad 3$

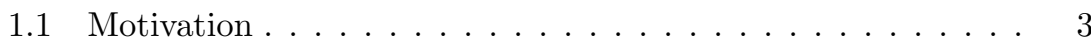

1.2 Problem formulation . . . . . . . . . . . . . . . . 8

1.3 Research method . . . . . . . . . . . . . . . . . 9

1.4 Contributions . . . . . . . . . . . . . . . . . . 10

1.5 Thesis outline ..................... 11

2 Background 13

2.1 Ontologies . . . . . . . . . . . . . . . . . 13

2.1 .1 Use of ontologies . . . . . . . . . . . . . 15

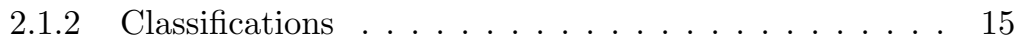

2.2 Description Logics . . . . . . . . . . . . . . . . . . . . . . . . . . . . . . 16

$2.2 .1 \mathcal{E} \mathcal{L}$ family . . . . . . . . . . . . . . . . 18

$2.2 .2 \quad \mathcal{A L C} \ldots \ldots \ldots \ldots \ldots \ldots$

2.3 Reasoning in description logics . . . . . . . . . . . . . . . . . . 20

2.4 Debugging and completing ontologies . . . . . . . . . . 23

2.5 Abduction in description logics . . . . . . . . . . . . . 24

2.6 Ontology matching . . . . . . . . . . . . . . . 26

3 Summary of papers $\quad 31$

4 Related work $\quad 35$

4.1 Completing the missing is-a structure . . . . . . . . . . . . 35

4.2 Detecting missing relations . . . . . . . . . . . . 36

4.3 Ontology matching . . . . . . . . . . . . . . . 39

4.4 Debugging semantic defects . . . . . . . . . . . . . . 42

4.5 Abductive reasoning in description logics . . . . . . . . . . 45

5 Conclusions and Future Work $\quad 49$

Bibliography $\quad 55$ 
II Papers $\quad 67$

Paper I Completing the is-a structure in light-weight ontologies

Paper II Get My Pizza Right: Repairing Missing is-a Relations in $\mathcal{A L C}$ Ontologies

Paper III Reducing the search space in ontology alignment using clustering techniques and topic identification

Paper IV Experiences from the Anatomy track in the Ontology Alignment Evaluation Initiative

Paper V User Validation in Ontology Alignment

Paper VI Integrating Ontology Debugging and Matching into the eXtreme Design Methodology 
Part I

Summary 



\section{Chapter 1}

\section{Introduction}

\subsection{Motivation}

The World Wide Web (WWW) is a network of web sites interconnected via hyperlinks. It is growing rapidly and as of May 2017 it is estimated to contain more than 1 billion web sites [2, 4]. Data on the WWW is available in different formats, such as documents, databases, images and videos. Often, this data has only limited structure. For example, web pages are often only semi-structured, containing just enough machine-readable meta-data for the correct presentation of a web site in a browser. The actual content (body) of web-pages is human-readable and often has no explicit structure.

The lack of structure makes the automation of more sophisticated queries - which require the understanding of the meaning of the data - a problem. As a result, large amounts of useful data on the WWW are not being used to their full potential. For example, querying for the age of a person in a document that contains the birth year of that person would already pose a difficulty for an automated agent. The agent would not have an understanding of the concept of age and how it relates to the birth year. In order to achieve queries like this, a preprocessing step such as knowledge extraction is often required. However, in many cases these preprocessing steps are incomplete and inaccurate and require human intervention to validate the extracted knowledge.

In some cases it may be necessary to combine information from multiple sources to answer a specific query. For example, in order to answer a query such as "Which actor from the movie Inception has the most Academy Award nominations?" we might have to access information on two separate web pages, one containing the cast of Inception and one with the list of all Academy Award nominees. To answer such queries it is necessary to navigate to multiple data sources and assemble the information. These data sources can be heterogeneous, having different data models or data in different formats, which would limit an automated agent's ability to answer 
such queries.

To deal with these issues Berners-Lee et al. [15] proposed the idea of a Semantic Web. It is supposed to be an extension of the WWW that would structure meaningful information on the Web, thus making it possible for automated agents to execute more sophisticated tasks. In order to do this, current human-readable content on the WWW has to be annotated with semantic labels which would be used by automated agents to extract meaning. Technologies used to achieve this include Extensible Markup Language (XML) and Resource Description Framework (RDF), which provide the necessary syntax for defining semantic labels, as well as a framework for defining statements about resources on the WWW. In addition, the vision of the Semantic Web is a Web of linked data where such annotated data is published and linked with other data on the Semantic Web.

The Semantic Web also provides support for modelling the domain of interest, i.e. describing which types of objects (i.e. concepts) exist, which kinds of properties they possess and how they relate to each other. This is done using ontologies, which provide the means for defining a formal vocabulary for a domain of interest. On top of this, ontologies also allow for inference and reasoning, which makes it possible to infer implicit knowledge from ontologies. Ontologies enable automated agents to acquire an understanding of the underlying data. They also provide a vocabulary for communication with other agents which can be used for data integration.

With the increase in popularity of the Semantic Web, more and more ontologies have been developed. Therefore, it is of no surprise that there are multiple ontologies for the same domains, with overlapping information. For example, there are a number of repositories for biomedical ontologies such as Open Biological and Biomedical Ontologies (OBO) Foundry, BioPortal, and Unified Medical Language System (UMLS). These ontologies are often used, for example, for annotating data resources, searching, or analysis of data. However, these ontologies were developed by different groups or organizations, with different applications for and points of view on the domain. In addition, the Semantic Web is decentralized and there are no naming standards when it comes to semantic labels. Therefore, two sources might use different labels for the same concept, which can cause problems when integrating information from multiple data sources.

Ontology matching attempts to solve this problem. The goal of ontology matching is to identify inter-ontology relationships. Knowledge of the inter-ontology relationships is important in many cases, for example in cases where it is necessary to use multiple ontologies, e.g., companies may want to use community standard ontologies in conjunction with company-specific ontologies. Other examples are integration, search and analysis of data in cases where different data sources in the same domain have been annotated with different but similar ontologies. The inter-ontology relationships known as mappings or correspondences define relations between entities in the ontologies (such as concepts, relations and instances). A set of map- 
pings (correspondences) is called an alignment. Ontologies together with the alignments between them form ontology networks. Finding the alignment between ontologies requires knowledge about the domain of ontologies, therefore making user intervention necessary. However, there are a number of ontology matching tools that can facilitate the process for the user by providing mapping suggestions which the user must then approve or reject.

Developing and aligning ontologies is not an easy task, and the resulting ontologies and ontology networks are often incorrect or incomplete which might lead to wrong conclusions being derived or valid conclusions being missed. Defects in ontologies and ontology networks can take different forms ranging from those that are easy to detect and resolve, such as syntactic defects that represent errors in syntax in the ontology representation, to more severe ones such as semantic and modelling defects. Semantic defects represent problems within the logic of the ontology, while examples of modelling defects are missing or wrong relations. Domain knowledge is required to detect and resolve modelling defects. In this work, we focus on incomplete ontologies and ontology networks, specifically ontologies with missing relations. In addition to being problematic for the correct modelling of a domain, incomplete ontologies also influence the quality of semantically-enabled applications.

When used in semantically-enabled applications, incomplete ontologies can lead to valid conclusions being missed. In ontology-based search, queries are refined and expanded by moving up and down the hierarchy of concepts. Incomplete structure in ontologies affects the quality of the search results. As an example, suppose we want to find articles in PubMed [5] using the Medical Subject Headings (MeSH) [3] term Scleral Diseases. PubMed is a database of abstracts primarily from the life sciences literature and MeSH is a thesaurus used for indexing PubMed records. By default the query will follow the hierarchy of MeSH and include more specific terms for searching, such as Scleritis. If the relation between Scleral Diseases and Scleritis were missing in MeSH, we would miss 1142 articles in the search result, which is about $59 \%$ of the original result ${ }^{1}$.

Incomplete alignment in an ontology network might also lead to incomplete results when, for example, an ontology network is used for integrating heterogeneous sources that are annotated using the ontologies in the network. Let us imagine that we have another data source that is annotated with the National Cancer Institute (NCI) Thesaurus and which we want to integrate with PubMed. One way to achieve this is by establishing an alignment between MeSH and NCI Thesaurus. For example, the NCI Thesaurus contains the concept Sclera Disorder, which corresponds to Scleral Diseases in MeSH. If this correspondence is included in the alignment then we could query PubMed for articles on scleral diseases using the concept Sclera Disorder from NCI Thesaurus, and conversely we could query our data source using the MeSH concept Scleral Diseases. However, if this correspondence

\footnotetext{
${ }^{1}$ PubMed accessed on 09-05-2017
} 
were missing then our query would not return any results (assuming that the correspondence is not implicitly derivable from the network).

Completing ontologies and ontology networks consists of two phases, detection and repair. In the detection phase missing relations are detected, and in the repairing phase the detected missing relations are made derivable in the ontology or ontology network. Ontology matching can be seen as a special case of ontology completion, where the inter-ontology relationships are the focus of the completion.

There are different ways to detect missing relations. One way is inspection by domain experts. Another way is using linguistic patterns, e.g. if we have concepts $\mathrm{X}$ and $\mathrm{Y}$ in the ontology and a statement " $\mathrm{X}$ such as $\mathrm{Y}$ " in some text, then a relation $\mathrm{Y}$ is-a $\mathrm{X}$ is a possible relation in the ontology. Ontology matching often utilizes string matching techniques, where concepts with similar labels are matched. Although there are many approaches for detecting missing relations, these approaches, in general, do not detect all missing relations. For instance, although the precision for the linguistic patterns approaches is high, their recall is usually very low.

In the following example, we discuss a process of completion of an ontology network that consists of two ontologies. Figure 1.1 shows parts of NCI Thesaurus and the Adult Mouse Anatomy (AMA) ontology concerning joints, which is relevant to our discussions. In order to establish an ontology network it is necessary to find the alignment between the two ontologies. For example, we can identify a number of correspondences between two ontologies, such as equivalence relations: Joint इ joint, Ankle Joint $\equiv$ ankle joint, Elbow Joint $\equiv$ elbow joint, Shoulder Joint $\equiv$ shoulder joint.

In addition, we can detect a number of missing relations in the AMA ontology. Let us assume that the detection phase of the completion of the AMA ontology yielded 6 missing is-a relations. Is-a relations (ㄷ) are relations between concepts which define that some concept is a subconcept (more specific) of some other concept e.g. Tree $\subseteq$ Plant. The detected missing is-a relations are: wrist joint $\sqsubseteq$ joint, hip joint $\sqsubseteq$ joint, knee joint $\sqsubseteq$ joint, elbow joint $\sqsubseteq$ joint, ankle joint $\sqsubseteq$ joint and shoulder joint $\sqsubseteq$ joint. In the ideal case, where our set of missing is-a relations contains all missing is-a relations, the repairing phase is easy. We just add all missing is-a relations to the ontology and a reasoner can compute all logical consequences. However, when the set of missing is-a relations does not contain all missing is-a relations - and this is the common case - there are different ways to repair the ontology. The missing is-a structure in the example can be repaired by adding limb joint 드 joint. This is-a relation is correct according to the domain and constitutes a new is-a relation that was not derivable from the ontology and was not originally detected by the detection algorithm. To illustrate why limb joint ᄃ joint repairs the detected missing is-a structure, consider the missing is-a relation wrist joint $\sqsubseteq$ joint. Since the relation wrist joint $\sqsubseteq$ limb joint is already derivable from the ontology, adding limb joint $\sqsubseteq$ joint would make wrist joint 5 joint derivable in the ontology. Similar reasoning holds for 


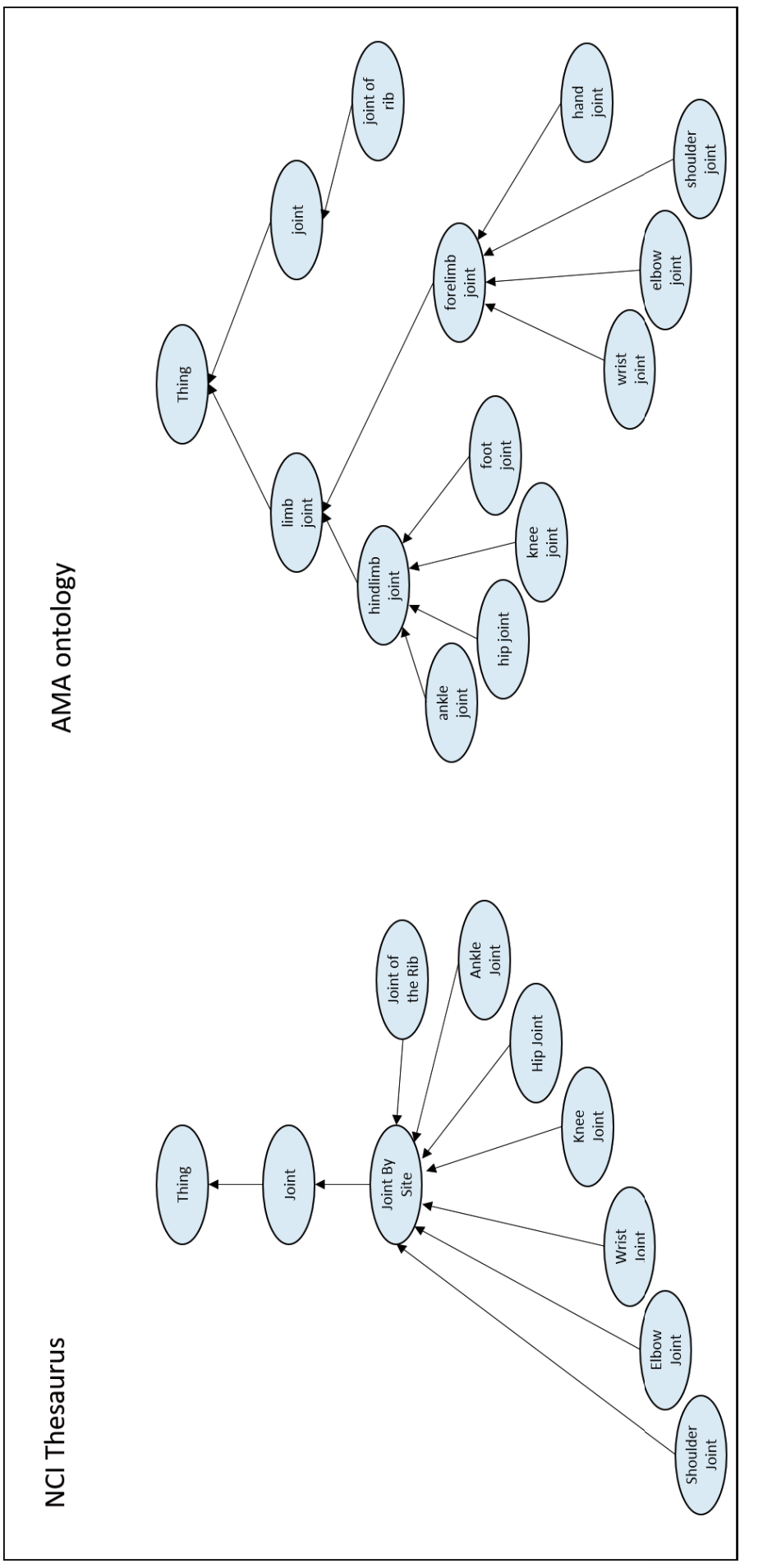

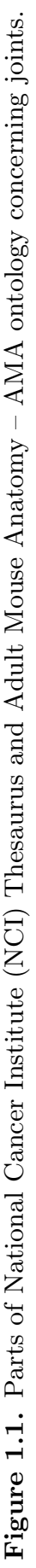


the other missing is-a relations in the set. We also note that from a logical point of view, adding limb joint $\sqsubseteq$ joint of rib also repairs the missing is-a structure. However, from the point of view of the domain, this solution is not correct. Therefore, as is the case for all approaches for dealing with modelling defects, a domain expert needs to validate the logical solutions.

\subsection{Problem formulation}

As the previous discussion pointed out, incomplete ontologies and ontology networks can lead to incomplete results in semantically-enabled applications. To deal with this problem it is necessary to detect and resolve missing relations in the ontologies and ontology networks. Therefore, the goal of this work is to address different issues in completing ontologies and ontology networks. The main research question is:

\section{How to complete ontologies and ontology networks?}

More specifically, the thesis addresses three aspects of completing ontologies and ontology networks, which are represented with three subsquestions:

1) How can the missing is-a structures and alignments in ontologies and ontology networks be completed?

In this work we focus on completing the is-a structures in ontologies and alignments. The is-a relation is the most common type of relation found in ontologies. For example, in the SNOMED Clinical Terms (SNOMED CT) [6] ontology, which is the largest collection of medical terms in the world with more than 300,000 concepts, is-a relations make up around one quarter of all statements about concepts and relations in the ontology. Equivalence correspondences between concepts are the most common type of correspondences currently supported by the majority of existing systems, as well as the most commonly evaluated type of correspondences at the Ontology Alignment Evaluation Initiative (OAEI), which is a yearly evaluation campaign for ontology matching systems.

We can divide the completing of ontologies and ontology networks into completing the ontologies and completing the relations between ontologies (i.e. alignment).

2) How to limit and facilitate user interaction in the completion process?

User validation is a necessary phase of the completion process, as only domain-correct relations should be added to ontologies and ontology networks. However, a user's time and effort are limited resources and therefore it is necessary to consider strategies and approaches which would both limit interaction with the user and facilitate the user's involvement. The relevance of user involvement is evidenced by the fact that nearly half of the future challenges of the ontology matching area [111] are directly related to it. 
3) How can the completion process be integrated into the ontology development phase?

While the completion of ontologies and ontology networks can be done at any point in the life-cycle of ontologies and ontology networks, some of the issues can be addressed early, in the development phase. While most methodologies for developing ontologies include a quality assurance step, very little existing work provides details on how this can be achieved. In a study [112] of larger ontology development projects it was found that while most of the projects used some form of methodology, quality checking and evaluation of the resulting ontologies was commonly omitted.

\subsection{Research method}

In the thesis we use a number of different research methods combining formal methods, implementation, and simulation. For all of the papers, a literature survey has been conducted in order to get a better overview of existing work related to the problem being studied. In Paper I and Paper II, which are concerned with completing the is-a structures in ontologies, we have used mathematical modelling to formalize the problem of completing the is-a structures in ontologies. We then proceeded by designing algorithms to solve the formalized problem. Some of the properties of the algorithms, such as soundness and completeness, were validated using mathematical proofs. Two tools were developed based on the designed algorithms. The development of the tools followed the prototyping software development methodology where a working version of the tool was developed, reviewed, and then further enhanced based on the review. The approach for completing ontologies was evaluated using a tool in an evaluation similar to a simulation, where we evaluated our methods in a controlled environment. The benefit of using simulations is a high level of precision, while its main drawback is limited realism. In order to increase the realism in our experiments we used real-world ontologies. Similarly, in Paper III we used simulation to evaluate our approach to reducing the search space.

We have conducted a case study related to the anatomy track of the OAEI in Paper IV. It analyzed the last 10 instances of the track. A case study is a qualitative research method which is used for gaining an in-depth understanding of the studied context as well as its dynamics [38]. The major disadvantage of the case study approach is that it is difficult to generalize. The case study research method was also used in Paper VI to evaluate our approach to integrating ontology matching and debugging into an ontology development methodology.

In Paper $\mathrm{V}$, in which we aim to identify requirements for user validation in ontology alignment, we have conducted a type of systematic literature review which was intended to identify the requirements. We have also conducted a number of simulations to measure the impact of user errors on on- 
tology alignment. The systematic literature review can be used to identify gaps in current research, summarize existing evidence of some phenomenon, or provide a framework or guidelines for new research [72]. The major advantage of a systematic literature review is that it gives an overview of the studied research question over a range of settings and empirical methods [72]. However, its drawback is that it requires more effort.

\subsection{Contributions}

The contributions of this thesis are as follows:

With respect to the question How can the missing is-a structures and alignments in ontologies and ontology networks be completed?

- We have formalized the problem of completing the is-a structures in ontologies as a generalized TBox abduction problem (GTAP) which is an extension of a TBox abduction problem [40]. Further, we introduced different preference criteria that are relevant to completing the is-a structure. These criteria also account for knowledge added to an ontology, in contrast to preference criteria in logic-based abduction, which usually emphasise only the solution size. We have developed algorithms for completing the is-a structures in more expressive ontologies. In this thesis we considered logic-based ontologies in the $\mathcal{E} \mathcal{L}$ family and $\mathcal{A L C}$, for which we developed two algorithms. We have developed two systems based on these algorithms and evaluated them against a number of ontologies with different levels of expressivity. In the experiments we have shown that our approach, in addition to repairing the ontology, also adds new knowledge that was not previously detected in the detection phase. While the approaches for completing is-a structures in ontologies discuss completion of individual ontologies, they are also applicable to ontology networks. In this case an ontology network can be treated as a single ontology and the discussed approaches would work across ontologies.

- With respect to the alignment, we have conducted an empirical study of the last 10 instances of the anatomy track and 2 instances of the anatomy task in the interactive track in OAEI. The study analyses the participating systems, the types of techniques used, and their performance. In addition, we have analyzed the general trends as well as common mistakes and rarely found correspondences.

- In order to address the problem of scalability of alignment algorithms, we have developed a method for reducing the search space when generating mapping suggestions. The method is based on clustering techniques. With this method we were able to generate partitions that allowed for high quality alignments with a highly reduced effort for computation of the parts of the ontologies in the partition. 
With respect to the question How to limit and facilitate user interaction in the completion process?

- We have conducted a qualitative study of the state-of-the-art ontology alignment systems to identify requirements for user validation in ontology alignment. The identified requirements pertain to three aspects of the user validation process: user, system services and user interface. In addition, we have also conducted experiments to analyse the impact of user errors on the ontology alignment process. While the requirements are discussed in the context of ontology alignment, they are directly applicable to the user validation phase in ontology completion.

- The methods that have been developed for reducing the search space when generating mapping suggestions in addition to reducing the computational effort for the parts of ontologies also impacts the user validation phase, as fewer mapping suggestions will be generated by the tool, thus requiring less user input for the validation.

With respect to the question How can the completion process be integrated into the ontology development phase?

- We have shown how both ontology completion and ontology matching can be integrated into a state-of-the-art ontology development methodology, thus addressing the issue of the quality of ontologies already in the development phase. In addition to completion, the proposed solution addresses other types of defects such as syntactic and semantic defects. The proposed approaches were evaluated in a case study based on a real-world ontology.

\subsection{Thesis outline}

The rest of the thesis is organized as follows:

Chapter 2 provides background on ontologies and description logics. In addition, the chapter discusses ontology debugging, ontology completion and ontology matching, and gives details on abductive reasoning in logic-based ontologies.

Chapter 3 gives a summary of the included works.

Chapter 4 covers an overview of related work with focus on completing ontologies and ontology networks.

Chapter 5 provides a discussion of the included works as well as directions for future work. 



\section{Chapter 2}

\section{Background}

This chapter presents background on areas that are relevant for this thesis. The chapter is organized as follows. First, in Section 2.1 we present the ontologies and discuss components, uses and a classification of ontologies. In Section 2.2 we provide some details about description logics and present variants of description logics relevant to this work. Reasoning in description logics is discussed in Section 2.3. The section introduces tableaux reasoning, which is an approach to reasoning in description logics that is used in this thesis. Details about different defects in ontologies are given in Section 2.4. Section 2.5 gives an overview of abduction problems in description logics and discusses preference criteria on solutions to abductive queries. Finally, in Section 2.6 we present an overview of the ontology matching process including the steps in the process and basic matching strategies.

\subsection{Ontologies}

The term ontology comes from philosophy, where it is the study of existence and the nature of being. It tries to answer questions such as "What does it mean to exist?" or "What can be said to exist?". In computer science the term was first used by McCarthy [92] in 1980 when discussing a new form of logic, where he suggested that ontologies can be used as a way of expressing commonsense knowledge. However, ontologies were still discussed in philosophical terms until the mid 80s when Alexander et al. [9] proposed a language for encoding ontological knowledge about the domain. This is recognized as the first use of the term ontology from a computer science perspective and a step away from philosophy [122]. Since then ontologies were adopted in many computer science communities, specifically in Artificial Intelligence, where ontologies became one of the important knowledge representation formalisms.

There are a number of definitions of ontologies in computer science. One of the first ones is by Neches et al. [94] which states: "An ontology defines 
the basic terms and relations comprising the vocabulary of a topic area as well as the rules for combining terms and relations to define extensions to the vocabulary". Probably the most cited definition in literature is by Gruber [49], where an ontology is defined as "an explicit specification of a conceptualization". Studer et al. [116] extended this definition and defined an ontology as "a formal, explicit specification of a shared conceptualization".

These definitions are related by the idea of conceptualization, i.e. an abstraction or a simplified view of the domain in question. The specification of this conceptualization should be explicit, i.e. the types of concepts, their relations and their use should be explicitly defined and formal, meaning that they are machine readable [116]. Studer et al. [116] also emphasized the need for this conceptualization to be "shared", meaning that it is the result of a consensus and does not only encode the knowledge of a single individual.

Ontologies differ in the kind of knowledge they can represent. Given this, different ontology components can be identified (e.g. [41, 76, 114]). Corcho et al. [24] define a minimal set of components that different kinds of ontologies share:

- Concepts (classes) - represent types of objects in the domain. Objects can be both abstract and concrete, as well as simple or complex, e.g. Man, Endocarditis, Carditis, PathologicalPhenomenon.

- Instances (individuals) - represent instantiations of concepts, i.e. actual objects, for example John. The assertion Man(John) represents that John is an instance of concept Man.

- Relations (properties, roles) - represent relations between concepts in the domain. Stevens et al. [114] define two types of relations:

- taxonomical - which represent relations that organize concepts into hierarchies. The two most used types of these are specialization relations (is-a, subconcept, subclass) and partitative relations (part-of). For example, Endocarditis is-a Carditis represents a specialization relation which defines that Endocarditis is a type of Carditis. An example of a partitative relation is the relation Lower jaw part-of Jaw.

- associative - which relate concepts across concept hierarchies (e.g. is-caused-by, has-associated-process, etc.).

- Axioms - model statements which are always true in a domain and which cannot be defined by other components [24]. Axioms are used to define such statements as cardinality restrictions (Man has exactly one Jaw), disjoint concepts (Endocarditis is not a Fracture) as well as general statements about the domain (e.g. Endocarditis is-a InflammatoryProcess and has-location Endocardium). These kinds of statements are useful for verifying if the knowledge in the ontology is consistent as well as for inferring new knowledge not explicitly defined in the ontology [24]. 


\subsubsection{Use of ontologies}

Ontologies have a number of uses, such as the following [75]:

- they are used as a means of communication between people and organizations;

- they enable knowledge reuse and sharing;

- they provide a basis for interoperability between systems;

- they are used for data integration;

- they are used as a repository of information.

In addition to being a key technology for the Semantic Web, ontologies are used in a variety of areas:

- Software Engineering - ontologies can be used in all phases of the software engineering life-cycle, e.g. as a means for representing different artefacts of a development process [55]. Ontologies are also used to support the systematic review process in Software Engineering [27];

- Artificial Intelligence - ontologies provide means for representing common sense knowledge [89];

- Computer Security - ontologies are used to encode properties of resources and different threats [58, 71];

- Biomedicine - ontologies are often used as knowledge repositories and as a means for data integration across heterogeneous data sources [96].

\subsubsection{Classifications}

Depending on the expressiveness of the knowledge representation formalism used for defining ontologies, a number of categories of ontologies can be defined. One of the first such classifications was introduced by Lassila and McGuinness [88] (later extended by Uschold and Gruninger [117]). This work defined an ontology spectrum which spans from inexpressive, lightweight ontologies represented in informal languages to very expressive ontologies represented in formal languages.

- Glossaries and Data Dictionaries - represent the simplest types of ontologies, essentially a list of terms. An example of this kind of ontology is a controlled vocabulary. In the case of glossaries, terms are associated with a meaning specified in natural language.

- Thesauri and taxonomies - represent ontologies that are lists of terms with a fixed set of relations between them. For example thesauri can define relations such as hyponym, antonym, synonym (e.g. WordNet $[8])$. In the case of taxonomies, terms are organized into an is-a hierarchy. 
- Ontologies represented using metadata, XML, schemas and data models - ontologies in this category can define concept hierarchies, attributes, relations and axioms.

- Ontologies represented using logical languages - represents the most expressive kind of ontologies based on a formal language (logic). The formal languages provide syntax and well-defined semantics as well as reasoning mechanisms such as consistency checking. Description logics is an example of a formal language widely used for defining ontologies.

A similar classification is given by Lambrix [75], where ontologies are classified based on the components and the information they contain.

\subsection{Description Logics}

Description logics is a family of formalisms used for representing knowledge in an application domain. In description logics an application domain is defined in terms of concepts that are used to describe entities in the domain. One of the main reasons for the popularity of description logics in knowledge representation systems is the emphasis on reasoning capabilities, which allow inference of implicit knowledge from explicitly defined descriptions.

There are three main building blocks in description logic languages [11]:

- Atomic concepts - unary predicates, representing types or sets of objects in the domain, e.g. Professor, Course, ResearchProject.

- Atomic roles - binary predicates, representing binary relations between the objects in the domain, e.g. teaches, worksOn.

- Individuals - constants, representing actual objects in the domain, e.g. john, mary, semanticweb101.

The vocabulary of a description logic language can be defined as a triplet $\left(N_{C}, N_{R}, N_{I}\right)$ where $N_{C}$ is a set of atomic concepts, $N_{R}$ is a set of atomic roles and $N_{I}$ is a set of individual names. Complex concept and role descriptions in the application domain are formed by combining basic building blocks and logical constructors such as conjunction $(\sqcap)$, disjunction $(\sqcup)$, existential quantification $(\exists)$, etc.

The semantics of concept descriptions are defined in terms of interpretations. An interpretation $\mathcal{I}$ consists of a non-empty set $\Delta^{\mathcal{I}}$ and an interpretation function ${ }^{\mathcal{I}}$, which assigns to each atomic concept $A \in N_{C}$ a subset $A^{\mathcal{I}} \subseteq \Delta^{\mathcal{I}}$, to each atomic role $r \in N_{R}$ a relation $r^{\mathcal{I}} \subseteq \Delta^{\mathcal{I}} \times \Delta^{\mathcal{I}}$, and to each individual name $a \in N_{I}$ an object $a^{\mathcal{I}} \in \Delta^{\mathcal{I}}$.

A knowledge base in description logics is an ordered pair $(\mathcal{T}, \mathcal{A})$ consisting of a terminological component called TBox $(\mathcal{T})$ and an assertional component called ABox $(\mathcal{A})$. 


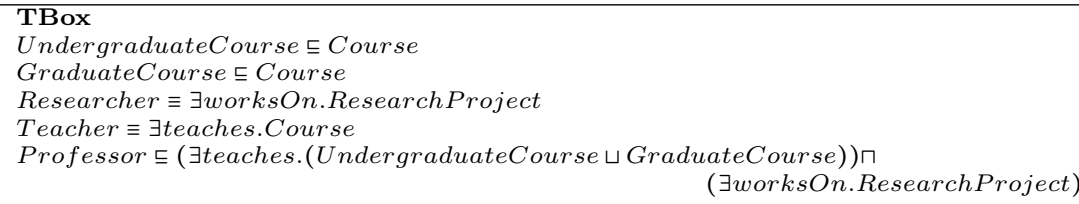

\section{ABox}

Professor $(j o h n)$

Course( sematicweb101)

teaches(john, semanticweb101)

Figure 2.1. A knowledge base - example.

A TBox contains a finite set of terminological axioms i.e. statements about how concepts and roles relate to each other. These axioms, in the general case, are of the form:

$$
\begin{aligned}
& C \sqsubseteq D(r \sqsubseteq s) \\
& C \equiv D(r \equiv s)
\end{aligned}
$$

where $C$ and $D$ are concepts (atomic or complex) and $r$ and $s$ are roles (atomic or complex) [11]. Axioms of the first type are called subsumption axioms (also known as inclusions, specializations, is-a relations). Regarding the semantics, an interpretation $\mathcal{I}$ satisfies a subsumption axiom $C$ 드 $(r \subseteq s)$ if it holds that $C^{\mathcal{I}} \subseteq D^{\mathcal{I}}\left(r^{\mathcal{I}} \subseteq r^{\mathcal{I}}\right)$. If an interpretation $\mathcal{I}$ satisfies an axiom (or set of axioms) then $\mathcal{I}$ is a model of this axiom (or a set of axioms). Axioms concerning concepts are also known as general concept inclusions (GCI) while axioms concerning roles are known as general role inclusions (GRI). Axioms of the second type are equivalence axioms. An interpretation $\mathcal{I}$ satisfies an equivalence $C \equiv D(r \equiv s)$ if it holds that $C^{\mathcal{I}}=D^{\mathcal{I}}\left(r^{\mathcal{I}}=s^{\mathcal{I}}\right)$. Equivalence $C \equiv D(r \equiv s)$ can also be represented with two subsumption axioms: $C \sqsubseteq D$ and $D \sqsubseteq C(r \sqsubseteq s$ and $s \sqsubseteq r)$. If the left hand side of an equivalence axiom is an atomic concept then these axioms are also known as concept definitions.

An ABox contains assertional knowledge, i.e. statements about the membership of individuals to concepts (concept assertions) and relations between individuals (role assertions). For example, Professor(john), Course( semanticweb101) are concept assertions and teaches(john, semanticweb101) is a role assertion where john and semanticweb101 are individuals, Professor and Course are atomic concepts and teaches is an atomic role. An interpretation $\mathcal{I}$ is a model of an ABox if for every concept assertion $C(a)$ it holds that $a^{\mathcal{I}} \in C^{\mathcal{I}}$ and for every role assertion $r(a, b)$ it holds that $\left(a^{\mathcal{I}}, b^{\mathcal{I}}\right) \in r^{\mathcal{I}}$. An interpretation is a model for a knowledge base if it is a model for the TBox and the ABox.

An example description logic knowledge base is given in Figure 2.1. In this example, Course, UndergraduateCourse, GraduateCourse, Teacher, ResearchProject, Researcher, and Professor are atomic concepts, teaches 
Table 2.1. The $\mathcal{E} \mathcal{L}$ family - Syntax and Semantics.

\begin{tabular}{|c|c|c|}
\hline Name & Syntax & Semantics \\
\hline \hline top & $\mathrm{T}$ & $\Delta^{\mathcal{I}}$ \\
\hline bottom & $\perp$ & $\varnothing$ \\
\hline nominal & $\{a\}$ & $\left\{a^{\mathcal{I}}\right\}$ \\
\hline conjunction & $C \sqcap D$ & $C^{\mathcal{I}} \cap D^{\mathcal{I}}$ \\
\hline $\begin{array}{c}\text { existential } \\
\text { restriction }\end{array}$ & $\exists r . C$ & $\begin{array}{c}\left\{x \in \Delta^{\mathcal{I}} \mid \exists y \in \Delta^{\mathcal{I}}:\right. \\
\left.(x, y) \in r^{\mathcal{I}} \wedge y \in C^{\mathcal{I}}\right\}\end{array}$ \\
\hline \hline GCI & $C \sqsubseteq D$ & $C^{\mathcal{I}} \subseteq D^{\mathcal{I}}$ \\
\hline equivalence axioms & $C \equiv D$ & $C^{\mathcal{I}}=D^{\mathcal{I}}$ \\
\hline RI & $r_{1} \circ \ldots \circ r_{k} \subseteq r$ & $r_{1}^{\mathcal{I}} \circ \ldots \circ r_{k}^{\mathcal{I}} \subseteq r^{\mathcal{I}}$ \\
\hline
\end{tabular}

and worksOn are atomic relations and john and semanticweb101 are individuals. The TBox contains three subsumption axioms, related to concepts UndergraduateCourse, GraduateCourse, and Professor, and two concept definitions (equivalence axioms) for concepts Teacher and Researcher. In natural language, the terminological axioms can be read as follows. Undergraduate course and graduate course are types of courses. A professor is someone who teaches some undergraduate or graduate course and works on a research project. However, not everyone who works on a research project and teaches such courses is a professor, therefore only the subsumption relation is used. Furthermore, teacher is defined as someone who teaches some course and a researcher is someone who works on some research project.

The ABox contains three assertions, two of which represent concept assertions, namely that john is a professor and that semanticweb101 is a course. Furthermore, the ABox also contains a role assertion which states that john teaches the semanticweb101 course.

As mentioned in the previous section, ontologies can be specified using description logics. In this case, concepts, relations, instances and axioms in ontologies map to concepts, roles, individuals and axioms in description logics, respectively.

There are different variants of description logics depending on which kind of logical constructors they allow. The supported logical constructors in a language have direct consequences on the properties of the language such as decidability, termination and completeness of reasoning. In this work we focus on two variants, the $\mathcal{E} \mathcal{L}$ family and $\mathcal{A L C}$.

\subsection{1 $\mathcal{E} \mathcal{L}$ family}

The $\mathcal{E} \mathcal{L}$ family of description logics includes three variants: $\mathcal{E} \mathcal{L}, \mathcal{E} \mathcal{L}^{+}$and $\mathcal{E} \mathcal{L}^{++}$. For the description logics $\mathcal{E} \mathcal{L}$ and $\mathcal{E} \mathcal{L}^{+}$the concept constructors are 
Table 2.2. $\mathcal{A L C}-$ Syntax and Semantics.

\begin{tabular}{|c|c|c|}
\hline Name & Syntax & Semantics \\
\hline \hline top & $\top$ & $\Delta^{\mathcal{I}}$ \\
\hline bottom & $\perp$ & $\varnothing$ \\
\hline conjunction & $C \sqcap D$ & $C^{\mathcal{I}} \cap D^{\mathcal{I}}$ \\
\hline disjunction & $C \sqcup D$ & $C^{\mathcal{I}} \cup D^{\mathcal{I}}$ \\
\hline concept negation & $\neg C$ & $\Delta^{\mathcal{I}} \backslash C^{\mathcal{I}}$ \\
\hline $\begin{array}{c}\text { existential } \\
\text { restriction }\end{array}$ & $\exists r . C$ & $\begin{array}{c}\left\{x \in \Delta^{\mathcal{I}} \mid \exists y \in \Delta^{\mathcal{I}}:\right. \\
\left.(x, y) \in r^{\mathcal{I}} \wedge y \in C^{\mathcal{I}}\right\}\end{array}$ \\
\hline universal \\
restriction & $\forall r . C$ & $\begin{array}{c}\left\{x \in \Delta^{\mathcal{I}} \mid \forall y \in \Delta^{\mathcal{I}}:\right. \\
\left.(x, y) \in r^{\mathcal{I}} \rightarrow y \in C^{\mathcal{I}}\right\}\end{array}$ \\
\hline \hline GCI & $C \sqsubseteq D$ & $C^{\mathcal{I} \subseteq D^{\mathcal{I}}}$ \\
\hline equivalence axioms & $C \equiv D$ & $C^{\mathcal{I}}=D^{\mathcal{I}}$ \\
\hline
\end{tabular}

the top concept $T$, conjunction, and existential restriction. For $\mathcal{E} \mathcal{L}^{++}$, we additionally have the bottom concept $\perp$, nominals, and a restricted form of concrete domains. In this thesis, we consider the version of $\mathcal{E} \mathcal{L}^{++}$without concrete domains. For the syntax and semantics of the different constructors see Table 2.1.

In $\mathcal{E} \mathcal{L}$, a TBox can contain two types of axioms: general concept inclusions of the form $C \sqsubseteq D$ (where $C$ and $D$ are $\mathcal{E} \mathcal{L}$ concepts) and equivalence axioms of the form $C \equiv D$. An equivalence axiom $C \equiv D$ can also be represented with two GCIs, $C \sqsubseteq D$ and $D \sqsubseteq C$.

In the case of $\mathcal{E} \mathcal{L}^{+}$and $\mathcal{E} \mathcal{L}^{++}$, TBoxes may also contain role inclusions (RIs) of the form $r_{1} \circ \ldots \circ r_{m} \sqsubseteq s$ (where $r_{i}$ and $s$ are role names).

\section{$2.2 .2 \quad \mathcal{A L C}$}

Description logic $\mathcal{A L C}$ was introduced by Schmidt-Schauß and Smolka [106]. The logical constructors in $\mathcal{A L C}$ are concept conjunction, disjunction, negation, and universal quantification. In the general case, description logic $\mathcal{A} \mathcal{L C}$ allows general concept inclusions of the form $C \sqsubseteq D$, where $C$ and $D$ are $\mathcal{A L C}$ concepts. The syntax and semantics of the logical constructors in $\mathcal{A L C}$ are given in Table 2.2.

In this thesis we consider ontologies that can be represented by a TBox that is an acyclic terminology. An acyclic terminology is a finite set of concept definitions (i.e. equivalence axioms of the form $C \equiv D$, where $C$ is an atomic concept) that contains neither multiple definitions nor cyclic definitions. A cyclic definition is a definition which defines concepts in terms of themselves or in terms of concepts that indirectly refer to them [11]. 


\subsection{Reasoning in description logics}

Knowledge bases usually contain implicit knowledge that is not explicitly defined using terminological or assertional axioms. In the example in Figure 2.1 it is easy to see that Professor is a Researcher, given that he/she works on a ResearchProject, and as a consequence john is also an instance of the concept Researcher. However, this knowledge is not explicitly defined in the knowledge base. In order to infer this implicit knowledge, knowledge representation systems based on description logics enable a number of reasoning tasks.

Reasoning tasks in description logics can be divided into two categories: reasoning tasks for concepts and reasoning tasks for ABoxes [11]. Reasoning tasks for concepts include checking [11]:

- Satisfiability - a concept $C$ is satisfiable w.r.t. a TBox T if there exists a model $\mathcal{I}$ of $\mathcal{T}$ such that $C^{\mathcal{I}}$ is non-empty. A TBox is said to be incoherent if it contains an unsatisfiable concept.

- Subsumption - a concept $C$ is subsumed by $D$ w.r.t. a TBox $\mathcal{T}$ if $C^{\mathcal{I}} \subseteq D^{\mathcal{I}}$ holds in every model $\mathcal{I}$ of $\mathcal{T}$. This can also be written as $\mathcal{T} \vDash C \sqsubseteq D$.

- Equivalence - a concept $C$ is equivalent to $D$ w.r.t. a $\operatorname{TBox} \mathcal{T}$ if $C^{\mathcal{I}}=$ $D^{\mathcal{I}}$ holds in every model $\mathcal{I}$ of $\mathcal{T}$.

- Disjointness - a concept $C$ is disjoint from concept $D$ w.r.t. a TBox $\mathcal{T}$ if $C^{\mathcal{I}} \cap D^{\mathcal{I}}=\varnothing$ holds in every model $\mathcal{I}$ of $\mathcal{T}$.

Reasoning tasks for ABoxes include the following tasks [11]:

- Instance checking - checking if an assertion $\alpha$ is entailed by an ABox $\mathcal{A}(\mathcal{A} \vDash \alpha)$, i.e. that every model of $\mathcal{A}$ is also a model of $\alpha$.

- Realization - given an individual $a$ and a set of concepts, the task is to identify the most specific concepts $C$ such that $\mathcal{A} \vDash C(a)$ where the most specific concepts are those that are minimal w.r.t. the subsumption ordering.

- Retrieval - represents retrieval of all individuals of some concept, i.e. for a given concept $C$ the idea is to identify all $a$ such that $\mathcal{A} \vDash C(a)$.

- Knowledge base consistency - a knowledge base is consistent if there exists an interpretation $\mathcal{I}$ that satisfies both $\mathcal{T}$ and $\mathcal{A}$.

The reasoning tasks are closely related and can often be reduced from one to the other. For example, a concept $C$ is subsumed by $D$ if $C \sqcap \neg D$ is unsatisfiable. Given this, reasoning algorithms usually provide the means for solving only one reasoning task, while the others are solved by reduction to it. 


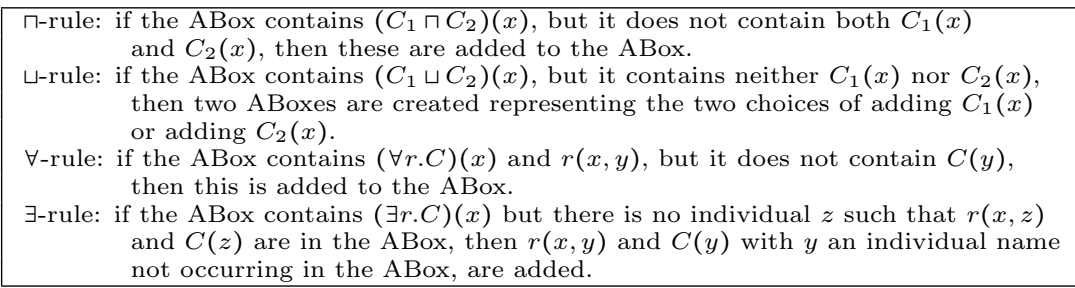

Figure 2.2. Transformation rules (e.g. [12]).

There are a number of reasoning algorithms for description logics, and in the following section we introduce the tableaux reasoning algorithm which is used in the thesis.

\section{Tableaux reasoning}

Checking satisfiability of concepts in ontologies represented in the studied description logics can be done using a tableau-based algorithm (e.g. [12]). To test whether a concept $C$ is satisfiable, such an algorithm starts with an ABox containing the statement $C(x)^{1}$ where $\mathrm{x}$ is a new individual. It is usually assumed that $C$ is normalized to negation normal form, i.e. negations can only appear in front of atomic concepts. This is done by applying De Morgan's laws and rules for quantifiers. For example, the negation normal form of $\neg(C \sqcup \exists r . D)$ would be $\neg C \sqcap \forall r . \neg D$. Next, consistency-preserving transformation rules are applied to the ABox. Figure 2.2 lists the rules for description logic $\mathcal{A L C}$. The $\sqcap-, \forall$ - and $\exists$-rules extend the ABox while the $\sqcup$-rule creates multiple ABoxes representing different choices for the disjunction. The algorithm continues applying these transformation rules to the ABox until no more rules apply. This process is called completion, and if one of the final ABoxes does not contain a contradiction - clash (we say that it is open), then satisfiability is proven, otherwise unsatisfiability is proven.

One way of implementing this approach is through completion graphs, which are directed graphs in which every node represents an ABox. Application of the $\sqcup$-rule produces new nodes with one statement each, while the other rules add statements to the node on which the rule is applied. The ABox for a node contains all the statements of the node as well as the statements of the nodes on the path to the root. Satisfiability is proven if at least one of the ABoxes connected to a leaf node does not contain a contradiction, otherwise unsatisfiability is proven.

In order to take subsumption axioms and concept definitions in the TBox into account, ABoxes have to be expanded with statements of the form $x: \neg C \sqcup D$ for every individual $x$ in the ABox, for each axiom $C \sqsubseteq D$ in

\footnotetext{
${ }^{1}$ These statements are also written as $x: C$.
} 


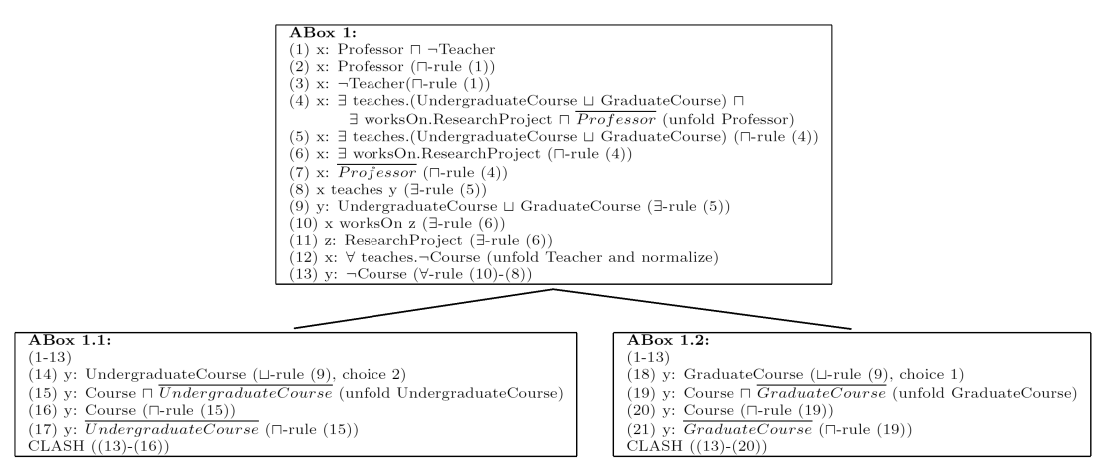

Figure 2.3. Completion graph for Professor $\square \neg$ Teacher.

the TBox. This is often a costly task, and different methods are used to minimize the need for such expansions.

In this thesis we assume that an ontology is represented by a knowledge base containing a TBox that is an acyclic terminology and an empty ABox. In this case reasoning can be reduced to reasoning without the TBox by unfolding the definitions. However, for efficiency reasons, instead of running the previously described satisfiability checking algorithm on an unfolded concept description, the unfolding is usually performed on demand within the satisifiability algorithm. When dealing with acyclic TBoxes, concept definitions are unfolded on demand as follows:

- If the TBox contains an axiom of the form $A \equiv B$ and an ABox contains a statement $x: A$ then statement $x: B$ is also added to the ABox.

- If the TBox contains an axiom of the form $A \sqsubseteq B$ and an ABox contains a statement $x: A$ then statements $x: B$ and $x: \bar{A}$, where $\bar{A}$ represents a new concept name, are also added to the ABox.

It has been proven that satisfiability checking w.r.t. acyclic terminologies is PSPACE-complete in $\mathcal{A L C}$ [90].

Figure 2.3 shows a completion graph for subsumption checking of the relation Professor $\subseteq$ Teacher with respect to the knowledge base in Figure 2.1. As explained earlier, the subsumption check can be reduced to a satisfiability check. Therefore, in order to prove that Professor $\subseteq$ Teacher holds, it is necessary to prove that Professor $\sqcap \neg$ Teacher is unsatisfiable on an empty ABox, meaning that all leaf ABoxes contain a contradiction. The algorithm starts with the statement $x$ : Professor $\square \neg$ Teacher where $x$ is a new individual. We continue by unfolding and applying $\sqcap-, \forall$ - and $\exists$-rules until no more unfoldings are possible and no more rules apply. In the completion graph in Figure 2.3 this is represented by steps (1) to (13) in ABox 1. Next, we apply a ப-rule which produces two new ABoxes containing statements from the initial ABox together with statements representing 
different choices for the disjunction (statements (14) and (18)). The algorithm continues applying transformation rules, and after adding statement (17) in ABox 1.1 a clash is detected given that $y$ is of type Course and $\neg$ Course at the same time. The same clash is detected in ABox 1.2. Given that all leaf ABoxes are closed the subsumption is proven.

\subsection{Debugging and completing ontologies}

With the increasing presence of data sources on the Internet, more and more research effort has been put into finding possible ways of integrating and searching such (often heterogeneous) sources. Semantic Web technologies such as ontologies are becoming a key technology in this effort. As discussed in Chapter 1, high quality ontologies and ontology networks are important for acquiring reliable results in semantically-enabled applications. However, it is often the case that defects are introduced into ontologies, both in the development phase and in updates. One of the reasons for this is that the domain experts who usually develop ontologies lack expertise when it comes to knowledge representation paradigms, and are often unaware of good and bad practices for developing ontologies. As a result, the ontologies they produce often have defects ranging from simple syntactic errors to wrong use of language constructs. For example, ontology developers often mistake the relation part-of for the $i s$ - $a$ relation. Another example of a defect is a situation in which domain experts introduce logical contradictions into the ontology.

In order to ensure high quality ontologies and ontology networks it is necessary to resolve these kinds of defects, which is the focus of ontology debugging and ontology completion. Ontology debugging and completing ontologies can be divided into two phases i.e. the detection phase and the repairing phase. In the detection phase, defects are detected using various techniques. The complexity of the detection phase varies with the types of defects.

In the repairing phase, the detected defects are repaired. The exact approach that is used to make the repair is dependent upon which kind of defects are debugged. For example, when dealing with ontology completion, i.e. missing relations, the idea is to add knowledge to the ontology that would make the missing relations derivable. One method for dealing with wrong relations is to remove relations that make the wrong relations derivable.

\section{Classification of defects}

There are three types of defects [66]:

- Syntactic defects - these represent syntactic errors, for example missing tags or incorrect format. This kind of defect is easy to detect and can be resolved using parsers and validators. 
- Semantic defects - these defects can be further classified into:

- unsatisfiable concepts - concepts to which no instance can belong, i.e. concepts that are equivalent to $\perp$. For example, let us consider an ontology with the following axioms:

\section{Bird $\sqsubseteq$ FlyingAnimal \\ Penguin $\sqsubseteq$ Bird $\sqcap \neg$ FlyingAnimal}

In this case concept Penguin is defined as a subconcept of Bird and a flightless animal ( $\neg$ FlyingAnimal). However, given that concept Bird is defined as a subconcept of FlyingAnimal it follows that Penguin is also a subconcept of FlyingAnimal. So in this case Penguin is at the same time a $\neg$ FlyingAnimal and a FlyingAnimal, which would mean that Penguin is equivalent to $\perp$ and therefore an unsatisfiable concept.

- incoherent ontologies - ontologies that contain an unsatisfiable concept. Therefore, the ontology from the previous example would be an incoherent ontology, given that it contains the unsatisfiable concept Penguin.

- inconsistent ontologies - ontologies which contain a contradiction, e.g. an instance of an unsatisfiable concept or an ontology from which it is possible to derive that $\perp \equiv T$. In our case, if we add an instance of concept Penguin to the ontology from the example it would be inconsistent.

As introduced in Section 2.3, one of the reasoning tasks in ontologies is satisfiability checking, which can be used to detect defects of this kind. However, the repairing phase is not trivial and there are a number of different approaches to dealing with defects of this type (see Chapter 4).

- Modeling defects - these represent defects that are a result of modeling errors. Examples of this kind of defect are missing and wrong relations. Missing relations are the focus of ontology completion. This kind of defect requires domain knowledge to detect and resolve. In Figure 1.1, examples of missing is-a relations in the AMA ontology are wrist joint $\sqsubseteq$ joint, hip joint 5 joint, knee joint 5 joint, elbow joint 5 joint, hindlimb joint $匚$ joint, forelimb joint $\sqsubseteq$ joint.

\subsection{Abduction in description logics}

Logical abductive reasoning is a type of inference. The task of abductive reasoning is, given a set of formulas (theory $T$ ) and a formula that represents an observation (an abductive query $O$ ), to find a set of formulas (an explanation $E$ ) such that $T \cup E$ is consistent and $T \cup E \vDash O$. In some definitions, 
logic-based abduction also includes a set of formulas $H$ called hypotheses, from which explanations are formed. When it comes to abductive reasoning in description logics Elsenbroich et al. [40] defined the following categories of abductive reasoning:

- ABox abduction - abductively retrieving concept or role instances which, together with the knowledge base, would entail a given ABox assertion.

- Concept abduction - abductively finding concepts that are subsumed by a given concept $C$.

- TBox abduction - abductively retrieving relations which, together with the knowledge base, entail a given relation $C \sqsubseteq D$.

- Knowledge-base abduction - abductively retrieving a set of TBox and ABox assertions which, together with the knowledge base, entail an abductive query that can be either an ABox or TBox assertion.

In this thesis we focus on TBox abduction, which is defined as follows.

Definition 1 (TBox Abduction [40]) Let $\mathcal{L}$ be a description logic, $\Gamma$ a knowledge base in $\mathcal{L}$, and $A, B$ concepts that are satisfiable w.r.t. to $\Gamma$ and such that $\Gamma \cup\{A \sqsubseteq B\}$ is consistent. A solution to the TBox abduction problem for $(\Gamma, A, B)$ is any finite set $S=\left\{E_{i} \sqsubseteq F_{i} \mid i \leq n\right\}$ of TBox assertions, such that $T \cup S$ is consistent and $T \cup S \vDash A \sqsubseteq B$. The set of all such solutions is denoted as $\mathcal{S}_{T}(\Gamma, A, B)$.

\section{Constraints on solutions}

Eiter and Gottlob [39] showed that computing all abductive solutions, even in propositional logic, is not possible or practical in all cases. Therefore, constraining solutions can significantly reduce the search space and allow practical use of logical-based abduction. Examples of constraints on solutions are subset minimality and minimum cardinality. A solution $S$ is said to be subset minimal if no proper subset of $S$ is a solution. In the case of minimum cardinality, solutions containing fewer formulas are preferred.

There are a number of restrictions that can be imposed on solutions of abductive problems in description logics. One such restriction is consistency, meaning that the union of the background theory (knowledge base) and the solution to the abduction problem should be consistent, e.g. $T \equiv \perp$ does not hold in the knowledge base. However, Elsenbroich et al. [40] argue that inconsistent solutions can be valuable, as they could imply the need for a revision of a knowledge base. Other restrictions such as relevance and minimality can be used for restricting trivial solutions. Relevant solutions are those solutions which do not directly entail the abductive query. In other words, an abductive query needs to be a logical consequence of a union of a solution and a knowledge base and not only the solution. Elsenbroich et al. 


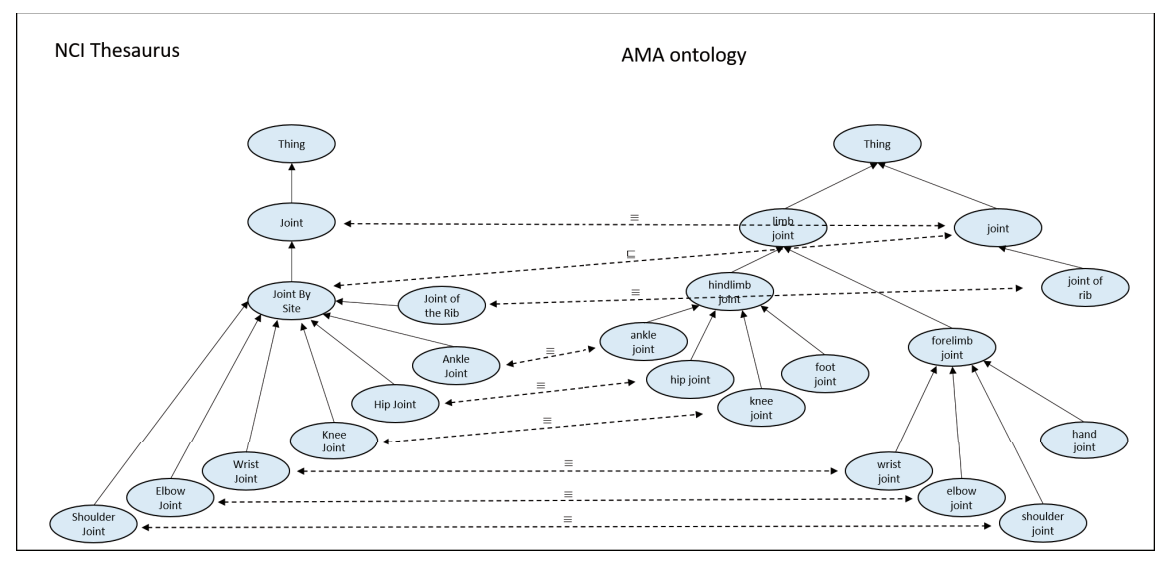

Figure 2.4. Example of an alignment between two ontologies.

[40] distinguish between two types of minimality: syntactic minimality, in which case a solution has to be of minimal length, and semantic minimality ${ }^{2}$, in which case a solution should only contain information which is necessary to make an abductive query a logical consequence of a knowledge base and a solution. For example, if $A$ is found to be a solution to some abductive query then $A \cap B$ is not a semantically minimal solution, as it contains $B$ which is extra information.

\subsection{Ontology matching}

Ontology matching or ontology alignment is a process of determining relationships between entities in ontologies, i.e. concepts, relations, instances and axioms. These relationships can be in the form of similarity values or as explicit relations such as equivalence, is-a or part-of relations. The inter-ontology relationships are called correspondences and the set of correspondences is called an alignment. The correspondences can be 1-to-1 (one-to-one) correspondences between entities in different ontologies, as well as 1-to-n (one-to-many), n-to-1 (many-to-one) and n-to-m (many-to-many) correspondences where multiple entities in one ontology are mapped to one or more entities in the other ontology. An ontology network is defined as a set of ontologies together with the alignments between these ontologies.

In Figure 2.4 we give an example of an alignment between the two ontologies introduced in Chapter 1. The correspondences are marked with dashed arrows. The alignment consists of 9 correspondences between concepts in two ontologies, 8 equivalence correspondences and a single is-a (subsump-

\footnotetext{
${ }^{2}$ This preference criterion is not directly related to semantic maximality, discussed later in this work.
} 


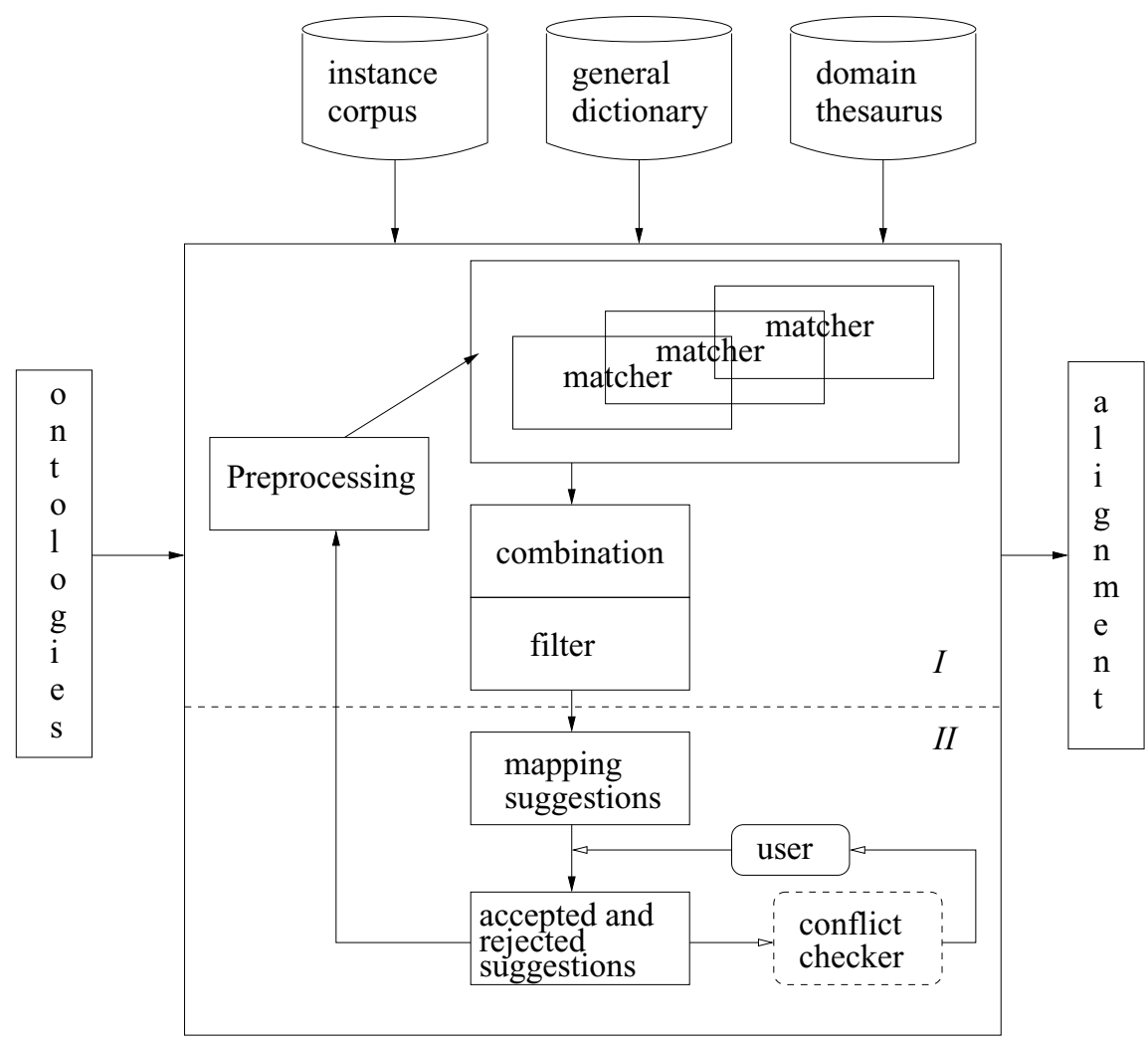

Figure 2.5. Ontology matching framework [80].

tion) correspondence. Most of the correspondences map one concept to a single concept in the other ontology, except in the case of concept joint in AMA which is mapped to two concepts in the NCI ontology, Joint via an equivalence relation and Joint By Site via an is-a relation.

Figure 2.5 gives an example of a general ontology matching framework that many existing ontology matching systems follow. The framework consists of two parts. In Part I the correspondences are computed and in Part II the computed correspondences are presented to the user for validation.

Part I of the matching process consists of the following three main steps:

Preprocessing. In the preprocessing step input ontologies are prepared for the alignment. This may include, for example, checking the validity of the files and feature selection to select only features which can be used by the algorithms. Another example of preprocessing is search space selection to reduce the search space so that there is no need to look at all possible pairs of entities being matched.

Matching. The goal of this step is to compute similarity values between 
entities from the input ontologies. The similarity values can be based on different aspects, such as linguistic properties or structure of the ontologies. We list the most common matching strategies:

- String-based strategies. The similarity between entities in the input ontologies is based on similarity between textual descriptions such as labels and definitions for relations, concepts or instances in the ontologies. For example, similarity can be defined as the number of matching contiguous sequences of $n$ letters in two strings ( $n$-gram). Another example would be defining the similarity between two strings as the minimum number of edits needed to transform one string to the other (edit distance).

- Structure-based strategies. These strategies take the structure of the ontologies into account. For example, two concepts can be considered to be more similar if the super-concepts or the sub-concepts of these concepts appear in an already-accepted correspondence.

- Constraint-based strategies. These strategies take into account different constraints on entities in two ontologies, such as data-types, cardinality, disjointness relation to other entities, etc. The more similar the constraints are, the more similar the entities are considered to be. For example, if two relations have similar data-types or similar cardinalities then these relations might be similar.

- Instance-based strategies. The similarity between two concepts is based on the number of shared instances of those two concepts.

- Use of auxiliary information. In some cases it may be possible to reuse external data sources as sources of domain knowledge for finding correspondences. For example, WordNet [8] can provide information about synonyms, hypo- and hypernyms of terms, and biomedical sources such as BioPortal [1], MeSH [3], and UMLS[7] can be used for matching biomedical ontologies.

Combining and Filtering. Most of the state-of-the-art systems allow combination of multiple matching strategies. Common approaches for combining results from multiple matchers include calculating the weighted-sum of the similarity scores from the selected matchers or selecting the maximum score among all of the matchers. Filtering is most commonly done using a threshold, i.e. correspondences with a similarity score above the defined threshold are accepted and passed to Part II of the process.

In Part II the correspondence suggestions from Part I are presented for validation to a user who makes a decision on their correctness with respect to the domain. Some ontology matching tools also provide different forms of conflict checking, with the aim of removing possible conflicts that the validated relations might cause in the ontologies. The matching process can be iterative, and the output from one iteration could be used as the input 
to the next. The benefit of this approach is that the validated relations can guide the matching process.

The increased interest from the research community in ontology matching has led to the creation of the Ontology Matching Workshop, as well as the Ontology Alignment Evaluation Initiative (OAEI). OAEI is an evaluation campaign with the aim of comparing and assessing the strengths and weaknesses of both ontology matching systems, as well as different matching techniques. In addition to this, OAEI has also aimed at improving evaluation techniques for ontology matching. It consists of a number of different tests covering different types of matching tasks such as instance matching, interactive matching, and matching ontologies in different languages. A large number of the tasks have a reference alignment which has been manually curated.

In cases where the gold standard (reference alignment) exists, measures such as precision, recall and F-measure can be used to evaluate the quality of the alignment. The precision of an alignment is the number of identified correspondences that are correct, i.e. those that appear in the reference alignment, divided by the total number of identified correspondences in the alignment. The recall is the number of identified correct correspondences divided by the total number of correspondences in the reference alignment. F-measure is defined using the following formula:

$$
\text { F-measure }_{\alpha}=(1+\alpha) \cdot \frac{\text { precision } \cdot \text { recall }}{\alpha \cdot \text { precision }+ \text { recall }}
$$

The $\alpha$ parameter can be used to assign different weights to precision and recall. Recall and precision have equal weights when $\alpha=1$. 



\section{Chapter 3}

\section{Summary of papers}

The chapter presents summaries of the included papers. The papers can be divided into three groups. The first group of papers focuses on completion of ontologies. More specifically, Paper I and Paper II address the issue of completing the is-a structures in ontologies with a focus on ontologies represented in description logics. The second group of papers deals with ontology matching. Paper III addresses the issue of scalability of ontology matching algorithms by proposing a method for reducing the search space. Paper IV provides a study of one of the longest running tracks at OAEI. Finally, Paper $\mathrm{V}$ looks at the problem of user validation and discusses different issues related to user-interaction during the ontology matching process. The third group includes Paper VI which addresses the issue of quality of ontologies by proposing a method for integrating ontology debugging and matching into the ontology development phase. Full papers can be found in Part II of the thesis.

\section{Paper I: Completing the is-a structure in light- weight ontologies}

The paper [87] considers the problem of completing the is-a structures in ontologies represented in the $\mathcal{E} \mathcal{L}$ family of description logics. The paper formalizes the problem as a generalized version of the TBox abduction problem (GTAP). We also discuss preference criteria for the solutions which, in addition to the size of a solution - a factor that is commonly addressed in abduction problems - also consider knowledge added to the ontology. The paper provides a complexity analysis for decision problems for the formalized problem, both with and without preference criteria. We have developed algorithms for solving GTAP which take as input a set of missing is-a relations and return a solution that is possibly more informative, thus adding new knowledge to the ontology which was not detected by detection algorithms. 
Therefore, our approaches can also be applied for detection of missing is-a relations. We have developed a system for repairing the missing is-a structure and showed the usefulness of our approach in two experiments dealing with real-world ontologies. In both cases, in addition to repairing the missing is-a structure, our approach has also identified a number of new missing is-a relations which were not in the initial set of missing is-a relations and which represent new knowledge added to the ontologies.

\section{Paper II: Get My Pizza Right: Repairing Miss- ing is-a Relations in $\mathcal{A L C}$ Ontologies}

In this paper (revision of [77]) we address the problem of completing the is-a structures in more expressive ontologies. The paper discusses an algorithm for solving GTAP in ontologies represented using $\mathcal{A L C}$ description logics. The algorithm for solving the problem is based on the tableaux reasoning algorithms. By finding ways of closing open branches in the tableaux we can produce solutions to the GTAP. The paper provides proofs for the soundness of our approach. We have also developed a system and conducted experiments using real-world ontologies. While the approach requires more time compared to the $\mathcal{E} \mathcal{L}$ system, the $\mathcal{A L C}$ approach is more general and is applicable to more expressive ontologies.

\section{Paper III: Reducing the search space in ontol- ogy alignment using clustering techniques and topic identification}

The paper (revision of [21]) addresses the issue of scalability in ontology matching. We propose an approach for reducing the search space when generating correspondence suggestions. The method presented in the paper is based on clustering techniques and text mining techniques such as topic identification. Clustering techniques are used to partition the ontology, while topic identification methods are used to identify topics of each partition, thus making it possible to find mappable parts. We have conducted experiments using the alignments from the anatomy track from the OAEI. The experiments have shown that our methods achieve high precision while significantly reducing the effort, i.e. the number of comparisons a matching algorithm needs to perform. This indirectly reduces the manual effort as the user is presented with fewer correspondence suggestions. However, other techniques will still be needed for finding correspondences that are not in the mappable parts. 


\section{Paper IV: Experiences from the Anatomy track in the Ontology Alignment Evaluation Initia- tive}

The paper [32] is a study of the last ten years of the anatomy track at the Ontology Alignment Evaluation Initiative, which is one of the longest running tracks of the campaign. First, we give details of the data sets used and summarize the evolution of the data set over the years. We then give an overview of all participating systems as well as the matching techniques they have used. We have conducted an analysis of the performance results for the period of 2007 to 2016, which gives an overview of the general trends. In addition, the paper provides an analysis of the most common mistakes as well as an analysis of the most commonly identified correspondences. This analysis has led to the identification of possible issues with the reference alignment and the ontologies. Finally, we propose possible changes and directions for the track.

\section{Paper V: User Validation in Ontology Align- ment}

The paper [31] addresses the issue of user validation in ontology matching, which is considered to be one of the challenges facing the ontology matching community. We have conducted a literature study in order to identify issues related to user validation in ontology matching. The identified issues are related to three aspects of user validation: the profile of the user, the services of the alignment system, and its user interface. The study also discusses how state-of-the-art matching systems deal with these issues. In addition to the study, the paper describes experiments to assess the impact of user errors in the validation process on the quality of the alignment, as well as how systems deal with errors.

\section{Paper VI: Integrating Ontology Debugging and Matching into the eXtreme Design Methodol- ogy}

While quality of ontologies is often addressed in the existing methodologies for developing ontologies, a description of the specific steps that need to be performed is often lacking. The paper [33] describes an approach for integrating ontology debugging and ontology matching into the process of ontology development. With respect to ontology debugging, we looked at syntactic, and semantic defects as well as the issue of ontology completion. The focus of our approach is the eXtreme Design (XD) methodology, which 
builds on the idea of reusing and specializing content patterns that cover certain modelling issues. We have conducted a case study on a real-world ontology developed using the XD methodology. The case study has shown that by integrating ontology debugging and matching it is possible to detect and repair a number of defects during the development phase, which would otherwise have ended up in the final ontology. 


\section{Chapter 4}

\section{Related work}

This chapter discusses related work on completing ontologies and ontology networks. We focus on the state-of-the-art work in completing the missing is-a structure and completing alignment, both of which were addressed in this thesis. In addition, the chapter gives an overview of existing methods for detection of missing is-a relations as well as other non-taxonomic relations that are related to the problem of ontology completion.

Our work on integrating ontology debugging and ontology matching into ontology development also addressed semantic defects in addition to the ontology completion problem. Therefore, in this chapter we provide an overview of existing methods for dealing with semantic defects. Finally, one of the contributions of the thesis is a formalization of the ontology completion problem as an abductive reasoning problem. In this chapter we look at related work on abductive reasoning in description logics.

\subsection{Completing the missing is-a structure}

There is not much work dealing with completing of missing is-a structures. This problem was addressed by Lambrix et al. [84] and Lambrix and Liu [83] in terms of taxonomies. Lambrix et al. [84] defined the problem of completing the is-a structure (called structural repairing). The problem can be seen as an abduction problem, however the authors' problem definition does not consider the role of a domain expert. The authors also introduced preference relations: axiom-preferred, information-preferred and strict-hierarchy-preferred. The axiom-preferred relation prefers repairs that do not contain redundant information, information-preferred prefers repairs that are more informative, and strict-hierarchy-preferred prefers repairs that do not change is-a relations in a taxonomy into equivalence relations. The solutions that are most axiom-preferred solutions would relate to a subset minimal solution in our setting. More informative solutions in our setting are a more strict version of information-preferred solution, since their work 
considers only is-a hierarchies. The authors introduced an algorithm for generating repairs based on Source and Target sets with respect to the introduced preference relations. An extension of the algorithm was proposed which considers the influences of repairs for other missing is-a relations when repairing a missing is-a relation. In both cases, the user can only select a single relation per missing is-a relation. The approach is non-iterative and there is no guarantee that the final solution is skyline optimal as it might not be subset minimal. The paper also discusses an approach for recommending repairing actions which aims to minimize interaction with background knowledge. The algorithms were implemented in a system which was evaluated on the AMA ontology and the NCI-A ontology.

The work by Lambrix et al. [84] focused on repairing a single ontology. Lambrix and Liu [83] extended the work by considering repairing missing is-a structure in taxonomies that are part of taxonomy networks, i.e. taxonomies networked by alignments. In addition to extending algorithms for generating, recommending and executing repair actions to deal with taxonomies in this setting, the extension also considers the problem of detecting missing is-a relations. In this case, candidate missing is-a relations are is-a relations which are derivable from a taxonomy network but are not derivable from a taxonomy itself. The candidate missing is-a relations must then be validated by a domain expert to acquire a final set of missing is-a relations.

We note that the approaches presented in this thesis for completing an is-a structure can be used for completing taxonomies, and in that case our approach finds more informative solutions than the previously described approaches.

The work by Lambrix and Ivanova [79] further extends the previous work and presents a unified approach for repairing missing and wrong is-a relations as well as missing and wrong mappings in networked taxonomies. The implemented system was evaluated against a number of taxonomies as well as in a use case [63].

Ivanova and Lambrix [61] presented a unified approach for aligning and repairing taxonomies. In this work, the authors have integrated their approach for repairing missing and wrong is-a relations and mappings with an alignment component based on the previous work by Lambrix and Tan [86]. The alignment component is used for detecting additional candidate missing mappings. The candidate missing mappings are then validated and validated missing mappings are passed to the repairing component for repair.

\subsection{Detecting missing relations}

There are different approaches for detecting missing is-a relations. A number of these approaches deal with detecting missing is-a relations in ontology networks. An approach by Bada and Hunter [13] discusses a method for enriching Open Biomedical Ontologies (OBO). This approach deals with subject-property-object terms in these ontologies and introduces the concept 
of nonalignments i.e. a subset of terms that have a subsumption relation between subjects but not between objects. This could imply that there are missing is-a relations between objects of terms in an nonalignment. Therefore, the next step is to use domain knowledge to determine if this is the case. If so, missing is-a relations need to be repaired. The authors propose two ways of repairing a nonalignment, either by adding is-a relations between objects or by removing is-a relations between subjects. As discussed in the previous section, a similar approach was used to detect missing is-a relations [83] and to detect both missing is-a relations and missing mappings in taxonomies [61, 63].

Bodenreider et al. [18] discuss and compare two different methods for aligning NCI-A and AMA ontologies, a lexical approach and a manual alignment. In the lexical approach, the first step is to identify mappings by matching pairs of concepts that match lexically. After that, a structural validation is executed which compares hierarchical relations among concepts in detected mappings across ontologies. In other words, if two concepts that appear in two mappings are related in one ontology then the concepts to which they map should also be related in the other ontology. If this is not the case, the mapping needs to be removed. Structural validation can also be used as a way of identifying candidate missing is-a relations. The pairs of concepts from mappings that cannot be structurally validated are candidate missing is-a relations.

Some approaches for detecting modeling defects focus on properties of the is-a relation. Guarino [50] suggests that the main reason that taxonomic information in ontologies is often complicated and difficult to understand is due to is-a overloading, i.e., linguistic definitions and properties of the is-a relation do not always reflect the ontological is-a relation. Therefore the author defines a number of ontology design principles which can be used to detect modeling defects in an ontology.

Keet [70] proposes methods for detecting and repairing flaws in object property expressions. Some examples of flaws are domain and range flaws, property characteristics flaws and property chain issues. The author defines two services for debugging these flaws, one dealing with property hierarchies and the other one dealing with property chains. The detection in these services is based on a number of tests that are run against property hierarchies and property chains. In addition, the author also specifies a number of guidelines on how to resolve situations where some test fails i.e. approaches for revising an ontology, which include changes in property expressions or class hierarchy.

Some of the ontology learning field's approaches to detecting relations between concepts in ontologies can be applied to detecting missing is-a relations. Ontology learning from text is discussed by Buitelaar et al. [20]. The authors cover a number of methods and applications for extracting ontology relations from text corpora. The methods in this area typically build upon established methods from computational linguistics and natural language 
processing.

There are methods in computational linguistics which specifically deal with the detection of is-a relations. The work done by Hearst [57] specifies a number of lexico-syntactic patterns for detecting hyponym relations in a text corpus. The specified patterns describe relationships between noun phrases. An example of a simple pattern is " $N P_{0}$ such as $N P_{1}$ and $N P_{2}$ " in which case $N P_{1}$ and $N P_{2}$ could be identified as hyponyms of $N P_{0}$. The author also describes a procedure for detecting new patterns where the idea is to first identify related terms given some lexical relation and then try to generalize parts of text where the detected related terms appear close to each other. While these types of patterns have a high precision, their recall is usually low.

Arnold and Rahm [10] propose three linguistically-based strategies for detecting relations between concepts in mappings. The first one, the compound strategy, suggests that a concept $A$ subsumes another concept $B$ if the name of $B$ is a compound word which ends with the name of $A$. The other strategy is based on background knowledge. Background knowledge sources such as thesauri and dictionaries are queried for linguistic relationships between concept names. The relationships are then used to decide relations between concepts in an ontology, e.g. synonym relation relates to equivalence, hyponym relation relates to subsumption. The third strategy is the itemization strategy which suggests that an is-a relation exists between an itemization and items that it contains.

Two approaches for ontology extension based on linguistic methods are presented by Wächter et al. [118]. The methods were illustrated on Gene Ontology (GO). They relied on having a selection of PubMed articles related to each term in the ontology. According to the first method, superstring prediction, terms which are superstrings of terms in the ontology, are possible candidates for including in the ontology. The second method, term co-occurrence analysis, takes co-occurrence of terms in a scientific text as an indication of possible candidate terms.

There are a number of methods for detecting relations between concepts in ontologies based on machine learning techniques and statistical methods.

Maedche and Staab [91] discuss methods for extracting non-taxonomic relations from text based on association analysis. The input to the association analysis is a set of concept pairs, which in turn are acquired from a text processing component which associates concepts based on their linguistic dependency or heuristic correlations, e.g. concepts are coupled if they appear in the same sentence or prepositional phrases are coupled with adjacent noun phrases.

A supervised classification-based method for aligning ontologies is presented by Spiliopoulos et al. [113]. The features used in the classification algorithm take into account both properties of textual descriptions of concepts (such as names, labels and comments) and properties of textual descriptions of related concepts, i.e., concepts related via equivalence, disjointness or sub- 
sumption axioms.

An approach for learning ontologies using probabilistic topic models is discussed by Zavitsanos et al. [123]. The approach uses Latent Dirichlet Allocation (LDA) [17] to identify topics from documents. The identified topics correspond to concepts in the new ontology. Conditional independence tests are then applied on the identified topics to detect taxonomic relations.

Cimiano et al. [22] present a method for learning taxonomic information from text based on Formal Concept Analysis (FCA) [46]. The inputs to the FCA are verb-subject, verb-object and verb-prepositional phrase pairs, which are acquired in a pre-processing phase. The output lattice from the FCA is transformed into a compacted partial order which corresponds to the resulting concept hierarchy.

Change management techniques from the ontology evolution area [56] can be used for detection of missing is-a relations. A process of adapting mappings in evolving ontologies is discussed by Dos Reis et al. [101]. The approach for adapting mappings is based on a categorization of ontology changes. The authors specified three algorithms that deal with mapping adaptation after removal, addition or revision of knowledge in an ontology.

\subsection{Ontology matching}

Ontology matching has been an active research area in the recent years. Interest within the community has lead to the creation of the Ontology Matching workshop, as well as the evaluation campaign OAEI, which have been run yearly since early 2000s. Together with other Semantic Web conference and workshops, these venues have been used to present a variety of approaches and systems for matching ontologies. There are a number of surveys on the state-of-the art (e.g. [65, 85, 95, 97, 109, 110, 111]) as well as books (e.g. [14, 41]) discussing different aspects of the field. However, even with more than 20 years of research there are still a number of challenges facing the community $[110,111]$. The first challenge is related to the issue of evaluating large-scale ontology matching. This kind of evaluation requires substantial manual effort to build high-quality reference alignments. In addition, new measures besides precision and recall are needed which would take into account the usefulness of a produced alignment. Next, three challenges are related to issues of efficiency of ontology matching. The first challenge in this group is related to the efficiency of matching techniques with respect to runtimes as well as memory requirements. Next, matching with background knowledge poses a challenge since most existing methods find new correspondences but many of the suggestions are wrong. Therefore, better strategies for reusing background knowledge with focus on precision are needed. Matcher selection, combination and tuning is not a trivial task and as more and more matchers are being developed more work will be needed on techniques for selecting, combining and tuning matchers to take full advantage of the aggregation of matchers. 
The next group of challenges is related to users of the systems. The first challenge is related to user involvement, i.e. systems need to provide decision support to the user in such a way that it is not overwhelming to the user. Interaction with the system needs to be natural and complete. More work is also needed on explanations of matching results. The explanations need to be concise, clear and precise. Allowing more people to vote and discuss correspondences can contribute to better alignment by lowering the impact of individual user errors. However, social and collaborative matching requires algorithms for ranking suggestions, voting strategies, and strategies to promote user participation.

Finally, the alignment management challenge relates to providing support and infrastructure for storing and sharing alignments.

Our work is related to a number of challenges. The work on reducing the search space for ontology matching relates directly to the challenge of efficiency of matching techniques. Shvaiko and Euzenat [111] suggest that the efficiency issues can be addressed by parallelization or distribution of matching tasks, approximation of matching results, modularization of ontologies or optimization of existing proven approaches. Our approach is based on modularization of ontologies. There have been a number of works $[29,59,81,107,120]$ which use the modularization of ontologies to limit the search space and number of similarity computations.

Seddiqui and Aono [107] propose the of the Anchor-Flood algorithm which segments the input ontologies with respect to anchors. An anchor is a pair of similar concepts in two ontologies. The segments are then built around anchors by iteratively adding neighbouring concepts (children and parents) to concepts in the segments. The alignment is then produced by aligning concepts in anchored segments in two ontologies.

Another approach based on anchors was proposed by Wang et al. [120]. However, this approach does not require computation of segments in ontologies. In this case anchors are used to find ignorable similarity computations based on the concept hierarchy and locality of matching, i.e., most elements of one part of the ontology would match elements in one part of the other ontology.

The method proposed by $\mathrm{Hu}$ et al. [59] computes ontology partitions using a variant of ROCK [51], exploiting a link measure based on structural similarities between concepts. After identifying partitions in ontologies they are assigned RDF sentences from the ontologies and possibly further extended in order to preserve RDF sentences within the block.

In the context of schema-matching Do and Rahm [29] propose a fragmentbased approach where the search space is reduced by matching non-overlapping subschemas. The matching is then done on similar subschemas which are, for example, those which have similar roots or context.

Other methods use the initial alignment to compute ontology partitions. Lambrix and Liu [81] use the initial alignment in the preprocessing step to partition the ontologies based on the structure of ontologies. In the GOMMA 
system [48] partitions are contain concepts which have a good coverage of concepts appearing in the initial set of correspondences.

The Malasco system [98] is a modular system implementing three different partitioning approaches. The first approach iterates over RDF sentences and creates partitions with $N$ triples. Other approaches reuse existing work on ontology partitioning presented by Stuckenschmidt and Klein [115] and Cuenca Grau et al. [26]. The author claims that the system achieved better precision when overlapping partitions were used.

Furthermore, in the thesis we focused on user validation, which relates to challenges of user involvement, explanation of matching results and social and collaborative matching. There have been earlier studies (e.g. [43, 44, 47, $62,78,42])$ addressing different aspects of user involvement in the matching process. These usually only focus on a subset of the three aspects identified in Paper V, i.e. the profile of the user, user interface and system services. In addition, we focus only on user validation in ontology matching and not user involvement in general.

One of the earliest evaluations of user interfaces was conducted by Lambrix and Edberg [78]. The authors have evaluated two state-of-the-art systems for ontology merging in bioinformatics. The evaluation consisted of two parts. The first part evaluated systems w.r.t. predefined criteria using a literature study. In the second part the user interface was evaluated through a user study with 8 users from different backgrounds to evaluate relevance, efficiency and learnability of the user interface, as well as users' attitude towards it.

The work by Falconer and Storey [44] proposes a theoretical framework for cognitive support in ontology matching. The theoretical framework is based on literature, existing work on cognitive support and decision making as well as on a case study conducted by the authors. The authors define a set of requirements for four conceptual dimensions, User Analysis and Decision Making, Interaction, Analysis and Generation and Representation. Later work [43] focuses on cognitive difficulties in ontology matching and surveys the existing visual ontology matching tools which involve the user in the matching task. The work has shown that most tools at that developed with findings from behavioural user studies in mind which made it difficult to identify possible features which would improve the interaction.

Granitzer et al. [47] provide a set of requirements for visual ontology matching systems. The requirements are divided into process driven and user driven requirements. Process driven requirements are related to different steps in the alignment process such as search for candidate correspondences or similarity computation. User driven correspondences are related to the use of processes for ontology matching.

Ivanova et al. [62] provide a set of requirements for fostering user involvement in large-scale ontology matching. The work builds on requirements from the earlier work $[44,47]$ which are further extended and refined to address the issue of user involvement in large-scale ontology matching. In 
addition, the authors have conducted a literature study as well as a heuristic evaluation and an observational study of state-of-the-art ontology matching systems to check the coverage of the requirements.

Euzenat and Shvaiko [42] give an overview of user involvement in the ontology matching process. The authors discuss interaction of individual users as well as interaction by sets of users, for example done through crowdsourcing or collaborative matching. In addition, the authors discuss different alignment explanation approaches and how they can facilitate user involvement in ontology matching.

\subsection{Debugging semantic defects}

Most of the work in debugging semantic defects aims at identifying and removing logical contradictions from an ontology. Standard reasoners are used to identify the existence of a contradiction, and provide support for resolving and eliminating it [45].

In works by Scholobach [104] and Schlobach and Cornet [105] minimal sets of axioms are identified which need to be removed to render an ontology coherent. This is done using the pinpointing algorithm. The idea behind this algorithm is that those axioms which are responsible for the incoherences should be removed with minimal intrusion in the ontology. In order to find these axioms, the algorithm first needs to find a minimal unsatisfiability-preserving sub-TBox (MUPS). A MUPS with respect to unsatisfiable concept A represents a minimal subset of TBox which contains A and is unsatisfiable. Generated MUPSs for all unsatisfiable concepts are used in the next step for the calculation of minimal incoherence preserving sub-TBoxes (MIPSs). MIPSs correspond to the minimal sets of axioms which cause the incoherence in the TBox. After finding the set of MIPS in the ontology, the decision regarding which axioms to remove has to be made. Therefore, Schlobach [104] introduces the concept of cores which represent sets of axioms that appear in one or more MIPSs. The number of MIPSs where some core appears represents an arity of a given core. The cores are then used by the algorithm to identify a set of pinpoints, i.e., axioms which need to be removed from an ontology to make it coherent.

Kalyanpur et al. [69] proposed two approaches for debugging unsatisfiable concepts, glass-box techniques and black-box techniques. The glass-box techniques can provide information about clashes (causes of defects) as well as a set of axioms which caused the clash. The clash information provides the user with information that is needed for debugging defects. The information usually includes names of concepts participating in the clash. The information about axioms participating in the clash is provided in the sets of support. These sets are formed during the execution of a reasoner. The reasoner needs to be extended in such way that it keeps extra information, which in the case of clash detection can be used to track the source axiom. The problems with the glass-box techniques are that they require reason- 
ers which are capable of generating explanations and the memory overhead needed for dependency tracking.

The black-box techniques do not have these requirements. The main idea of black-box techniques is to divide unsatisfiable concepts into root concepts (those which are not dependent on any other unsatisfiable concept) and derived concepts (unsatisfiable concepts dependent on some other unsatisfiable concepts). The algorithm for searching for root/derived concepts is divided into two steps: detection of asserted dependencies and detection of inferred dependencies. The first step focuses on finding dependencies between unsatisfiable concepts among assertions within the ontology while the detection of inferred dependencies searches for dependencies among inferred structures of the ontology.

However, the presented techniques only provide the user with information which helps the user in understanding defects in an ontology. The actual repairing is done manually. Kalyanpur et al. [68] extended their work, proposing an approach to debugging unsatisfiable concepts similar to pinpointing $[104,105]$. Their algorithm is based on precise MUPS, an extension of MUPS, which describes the specific parts of an axiom that make the axiom unsatisfiable. To get the precise MUPS, the search for the MUPS is done on a new knowledge base which is made by splitting axioms across conjunctions into shorter and simpler axioms. The axioms that are considered suitable for removal are acquired using a modified version of the hitting set algorithm [102], which takes into account a ranking of an axiom. The authors present a number of different ranking approaches, such as impact of removal, annotation information, number of times an axiom appears in an ontology, etc. In order to optimize the repairing process the authors reused the idea of root and derived unsatisfiable concepts [69]. The idea is that by repairing the root concepts a large portion of unsatisfiable concepts that were dependent on these concepts would also be repaired.

In the work by Haase and Stojanovic [52] the focus is on maintaining consistency as the ontology evolves. This is based on a formalization of the semantics of change for ontologies. Three types of consistency are formally defined: structural consistency, logical consistency, and user-defined consistency. The authors also discuss resolution strategies for each type of corresponding inconsistency. In the case of logical consistency, two alternatives for resolution are discussed. The first alternative is to identify a consistent ontology for a given change by removing one axiom at a time from an inconsistent ontology. The second alternative suggests that the resolution should be done by localizing the inconsistency i.e. identifying a minimal set of axioms that make the ontology inconsistent.

In works by Meilicke et al. [93] and Ji et al. [64] the setting is extended to repairing ontologies connected by mappings. In this case, semantic defects may be introduced by integrating ontologies. Meilicke et al. [93] address the problem of finding and repairing incoherences caused by the automatic mapping process. The proposed debugging algorithm is based on conflict 
sets. Conflict sets extend the idea of diagnosis [102] where, given a set of components $C O M P$ and a set of observations $O B S$, the task is to identify a subset of erroneous components so that the remaining components are consistent. In the case of distributed ontologies, a set of components COMP represents a set of mappings between ontologies and a set of observations $O B S$ is a set of concept subsumptions in the ontologies. The proposed algorithm returns minimal conflict sets, i.e., conflict sets whose subsets are not conflict sets. Thus, repairing one component from the minimal conflict set would repair the conflict. The decision on which mappings should be removed from the minimal conflict steps is based on the confidence value of each mapping, if specified, or on the WordNet [8] distances between concepts participating in the mapping.

Ji et al. [64] propose a relevance-directed approach for diagnosing and repairing ontology networks. Their approach is based on computing MUPS between sub-ontologies and is adapted from the algorithm proposed by Kalyanpur et al. [67], which is based on the hitting set algorithm [102]. The approach is relevance-directed as there are 3 strategies for computing MUPS: computing a single MUPS, computing some MUPS and some hitting sets, and computing all MUPS and all hitting sets. The decision on which strategy to use is made by a user given an ontology and his/her purpose.

Most approaches for debugging semantic defects return a list of possible diagnoses that can repair the ontology. However, not all of these are desired by the user. Shchekotykhin et al. [108] propose query strategies for interactive debugging of ontologies which, through a series of queries to the user, aim to identify the target diagnosis.

The work by Qi et al. [100] further characterizes the problem as mapping revision. The authors define a conflict-based mapping revision operator based on minimal conflict sets [93]. Using belief revision theory, the authors give an analysis for the logical properties of the revision algorithms.

Another approach for debugging mappings is proposed by Wang and $\mathrm{Xu}$ [119]. In this work, the authors define four types of defects in mappings, i.e. redundant mappings, imprecise mappings, inconsistent mappings and abnormal mappings. In addition, algorithms for detecting and diagnosing each type of defined defects are defined. The algorithms output warnings about detected defects as well as suggestions on how to debug them. The users have to manually execute the suggestions.

The work by Corcho et al. [25] deals with both modeling and semantic defects. The authors propose an approach for detecting modeling and semantic defects within an ontology based on patterns and antipatterns. The proposed patterns and antipatterns contain those patterns which have an impact on logical consequences and those which are supposed to improve the intended meaning of ontologies and their understandability. In addition to defining patterns and antipatterns, the authors also propose ways of solving the issues detected by the patterns and antipatterns. The work specifies a debugging strategy which defines the order in which a user should resolve 
detected patterns and antipatterns.

Roussey and Zamazal [103] presented a method for detecting, without a reasoner, some of the antipatterns introduced by Corcho et al. [25]. The antipatterns are detected by running a SPARQL query on a transformed ontology. A transformed ontology is acquired from the original ontology by applying transformation rules that are designed to simplify class definitions, harmonize different implementation styles, and simulate inferences, thus avoiding the need for a reasoner.

Another work by Lambrix and Liu [82] considers both modeling and semantic defects, where the authors provide a method for repairing wrong missing is-a structure and wrong mappings in ontology networks. In addition to modeling defects, this method uses approaches for debugging semantic defects.

Poveda-Villalón et al. [99] present a web-based tool for validating ontologies against a catalogue of defined pitfalls. The pitfalls cover different categories of issues. These categories have to do with issues that are related to syntactic correctness of the ontology with respect to language specifications, documentation and clarity of the code, logical consistency, and modelling issues.

\subsection{Abductive reasoning in description logics}

Elsenbroich et al. [40] defined four different abductive reasoning tasks concept abduction, ABox abduction, TBox abduction and knowledge base abduction.

Most existing approaches for description logic abduction focus on ABox and concept abduction and are mostly based on existing proof techniques such as semantic tableaux and resolution. Since the number of possible solutions can be infinite, these approaches introduce constraints on solutions.

Halland and Britz [53] propose a semantic tableaux approach for ABox abduction in $\mathcal{A L C}$. In their approach, the authors perform instance checking on an abductive query. After extracting a full completion graph, the algorithm generates a set of concept assertions for each open branch which would close that branch. This is done by taking negations of concept assertions in open branches. The solutions to the abductive query are then acquired by running the minimal hitting set algorithm [102] on these sets. This algorithm is sound but not complete and the solutions are not guaranteed to be semantically minimal.

The work by Klarman et al. [73] proposes both a semantic-tableaux and a resolution based approach for ABox abduction. The proposed approaches are goal-oriented, meaning that only actions which contribute to the solution are chosen in the proof procedures. This is done by using regular connection tableaux for the tableau-based approach or sets-of-support in the case of resolution proofs. Both approaches, however, require translation to first order logic (conjunctive normal form) and the solutions have to be translated 
back to description logic. The method is both complete and sound for consistent and semantically minimal solutions. Since the set of solutions can contain some inconsistent and non-minimal solutions additional checks are required to guarantee consistency and minimality.

A practical approach for ABox abduction, based on abductive logic programming, was proposed by Du et al. [34]. This approach considers solutions over a finite set of concepts and roles called abducibles, which is in contrast to the work by Klarman et al. [73] where solutions can be formed using arbitrary concepts which can lead to infinitely many solutions when dealing with cyclic definitions. The proposed approach [34] uses existing abductive logic programming systems and therefore requires a transformation to a plain Datalog program. The solutions are consistent and minimal given a set of abducibles. In this case, allowed abducibles are atomic concepts or atomic roles. The approach does not guarantee completeness since the translation to a Datalog program is approximate and in some cases a solution would not be found. The authors extended their work [35] by allowing arbitrary concepts and roles as abducibles, such as complex concepts, negated roles, etc. This work was further extended [37] to deal with an ABox abduction problem where new individuals are allowed in solutions. For example, for abductive query Person(Tom) in the case where the ontology contains $\exists$ hasParent.Person $ᄃ$ Person an intuitive minimal explanation is a set $\{$ hasParent $($ Tom,$u)$, Person $(u)\}$ where $u$ is a new individual. As a consequence of this extension there can be infinitely many minimal solutions. To deal with this, the authors reduce the number of explanations which need to be computed by considering only representative solutions, that is solutions which are not subsumed by other minimal solutions. This approach was compared to the approach by Du et al. [34] in an experimental evaluation, which has shown that computing representative solutions is more efficient than computing minimal solutions.

There are a number of approaches which deal with the concept abduction problem as well as the conditionalised concept abduction problem, where the idea is for concepts $C$ and $D$ and a TBox $T$ to identify a concept $H$ such that $C \sqcap H \sqsubseteq D$ holds in $T$ and $C \sqcap H \nsubseteq \perp$.

Colucci et al. [23] consider an approach to the conditionalized concept abduction problem. The approach they present uses a variation of the semantic tableaux and also proposes two labelling functions. The two labelling functions $T()$ and $F()$ represent true and false formulas in a tableaux. The solutions are formed from concepts which have at least one constraint in $\mathrm{F}($ ) of every open branch. This choice is non-deterministic and can be used to select solutions based on some criteria. The algorithm also contains a consistency check, which implies that the produced solutions are always consistent.

Another approach to conditionalized concept abduction in $\mathcal{A L N}$ description logics was proposed by Di Noia et al. [28]. This approach operates on concepts in conjunctive normal form and is based on a modified version of 
the structural subsumption algorithm [19]. A structural subsumption algorithm is used to identify concepts $H$, which need to be conjoined with $C$ so that $C \sqcap H \sqsubseteq D$ holds. The proposed algorithm returns irreducible solutions, i.e. solutions which are minimal conjunctions.

The complexity of concept abduction in the $\mathcal{E} \mathcal{L}$ family of description logics is explored by Bienvenu [16]. The abduction problem discussed in the paper includes a set of possible hypotheses. The work focuses on three decision problems, i.e. existence (does a solution exist), relevance (does the hypothesis appear in some solution) and necessity (does the hypothesis appear in all solutions). The complexity of the decision problems is analysed in terms of a number of preference criteria, such as subset minimality, minimal cardinality and minimal weight. The analysis has shown that the complexity of concept abduction for the decision problems is equal to the complexity of abduction in propositional definite Horn theories in the case of $\mathcal{E} \mathcal{L}$ and $\mathcal{E} \mathcal{L}^{+}$. In the case of $\mathcal{E} \mathcal{L}^{++}$the complexity is the same as in the case of propositional Horn theories.

There has not been much work related to TBox abduction, which is the most relevant abduction problem for this thesis. The work by Hubauer et al. [60] proposes an automata-based approach to TBox abduction using abducibles. It is based on a reduction to the axiom pinpointing problem which is then solved with automata-based methods. Similarly to the approach presented by $\mathrm{Du}$ et al. [34], the abductive problem also contains a set of abducibles which in this case represent a set of axioms that can appear in solutions.

A recent approach by Halland et al. [54] considers the TBox abduction problem in $\mathcal{A L C}$ ontologies. The presented approach is based on tableaux reasoning, where the solutions to the abductive query are acquired by generating a set of axioms for each open branch in the full completion graph such that when the axioms are added to the knowledge base, it would close the open branch. The axioms which close open branches are of the form $\mathrm{C}$ $\subseteq \mathrm{D}$, where $\mathrm{C}$ is of the form $A, \neg A$ or $\exists r . T$ and $\mathrm{D}$ is of the form $A, \neg A$ or $\forall r$.T where $A$ is an atomic concept and $r$ an atomic role. Similarly to their previous approach [53] the authors use the minimal hitting set algorithm [102] to extract a solution from these sets of axioms.

The authors have also shown that their approach for solving a TBox abduction problem can be applied to the generalized version of a TBox abduction problem, which considers multiple subsumption axioms in the abductive query. In this case for an abductive query $\left\{C_{1} \sqsubseteq D_{1}, \ldots, C_{n} \sqsubseteq D_{n}\right\}$ the satisfiability checker needs to be run on input $\left(C_{1} \sqcap \neg D_{1}\right) \sqcup \cdots \sqcup\left(C_{n} \sqcap \neg D_{n}\right)$.

Both the approach for the TBox abduction problem and the generalized version of a TBox abduction problem require a post-processing step to test for semantic minimality, consistency and relevance. The approaches are sound and complete with respect to the allowed constructors for relations in the solution.

The presented approaches for description logic abduction mostly deal 
with relatively inexpressive ontologies, such as $\mathcal{E} \mathcal{L}$ and $\mathcal{A L C}$. However, the work by Donini et al. [30] describes a type of conditionalised concept abduction called structural abduction which is applicable to $\mathcal{S H}$. In this case, instead of finding concept $H$ such that $C \sqcap H \sqsubseteq D$, the presented algorithm first finds places within a description of $C$ where hypotheses variables can be added. Then, a solution is defined as a substitution of a set of variables with concepts which cause the new extended concept to be subsumed by $D$. A more recent approach [74] describes an approach using saturation-based reasoning for concept forgetting. The TBox abduction is defined as a form of concept forgetting problem and the solution contains only concepts appearing in the signature of abducibles. The approach is implemented for $\mathcal{A L C H}$.

The work by Du et al. [36] presents an approach for TBox abduction based on justification patterns. A justification pattern is acquired by introducing variables instead of concepts' names (respectively roles) into justifications for some concept inclusion. The axioms in the solution can contain arbitrary DL constructors.

The computational complexity of the generalized TBox abduction problem in the $\mathcal{E} \mathcal{L}$ family of description logics was analysed by Wei-Kleiner et al. [121]. The analysis focused on three decision problems: existence (does a solution exist), relevance (does a set of axioms appear in some solution) and necessity (does a set of axioms appear in every solution). These problems relate to the problems discussed by Bienvenu [16], with the difference being that, rather than including a set of hypotheses our abduction problem includes an oracle function which returns true or false depending on whether or not an is-a relation is correct according to the domain. The computational complexity of these problems was explored with respect to the preference criteria presented in this thesis. The analysis has shown that skyline optimal solutions are the best choice in practice. 


\section{Chapter 5}

\section{Conclusions and Future Work}

The World Wide Web (WWW) is growing rapidly. However, data on the WWW often has only limited structure. This limits the opportunities for automation of queries that require an understanding of the meaning of the underlying data, as well as queries which require data from multiple sources. As a consequence large amounts of useful data are not being used to their full potential. A step towards support for queries of this kind is the idea of the Semantic Web, which represents an extension of the current WWW. The Semantic Web aims at structuring the data, thus adding meaning to it, which would allow automated agents to execute more sophisticated queries.

Ontologies are one of the key technologies in this endeavour. Ontologies provide a means for modelling a domain of interest i.e. establishing a vocabulary of the domain. Ontologies and alignments between them can be used for data integration and as a basis for data interoperability.

However, ontologies and ontology networks are often incomplete, a consequence of which is that semantically-enabled applications that use such ontologies and ontology networks might omit relevant and correct results. Therefore, there is a need for high quality ontologies and alignments.

The focus of this thesis has been completion of ontologies and ontology networks. With respect to completing the ontologies, our work focused on is-a relations in ontologies, which are the most common type of relations found in ontologies. We considered ontologies represented in description logics, more specifically the $\mathcal{E} \mathcal{L}$ family and $\mathcal{A L C}$ family of description logics. The $\mathcal{E} \mathcal{L}$ family of description logics is highly relevant for the representation of lightweight ontologies and some of the major biomedical ontologies are represented in $\mathcal{E} \mathcal{L}$. On the other hand, $\mathcal{A L C}$ is often considered a basic expressive description logic containing logical constructors such as disjunction, existential quantifier and universal quantifier, which sets a foundation for more expressive description logics. 
We formalized the problem of completing the is-a structures in ontologies as a generalized version of the TBox abduction problem. In contrast to logic-based abduction where the idea is to identify solutions that are as small as possible, the goal of repairing missing is-a structure is to add more subsumptions to enrich the ontology. Therefore, our formalization also includes a preference criterion which emphasizes the informativeness, i.e. the knowledge added to the ontology. We have also developed algorithms for solving the GTAP in the $\mathcal{E} \mathcal{L}$ family and $\mathcal{A L C}$ description logics. The $\mathcal{E} \mathcal{L}$ algorithm relies on identifying logical patterns in normalized $\mathcal{E} \mathcal{L}$ ontologies for extracting solutions to GTAP. In this case, the reasoner is used as a black box and the logical patterns are applied to the result of the reasoning process. On the other hand, the $\mathcal{A L C}$ algorithm is more general and is based on satisfiability checking using tableaux reasoning. This approach is a glass-box approach, as the logical patterns used to identify solutions to the GTAP are applied directly to the completion graph of a tableaux reasoner. The algorithms are iterative and in every iteration, except for the last, a more informative solution is acquired. We have implemented systems for completing $\mathcal{E} \mathcal{L}$ and $\mathcal{A L C}$ ontologies based on our algorithms. The analysis and the experiments have shown that our approach for completing the is-a structures in ontologies is useful, as in the experiments we have identified a number of new is-a relations which were not detected by the detection algorithm. The iterative approach has also proven to be advantageous, as in subsequent iterations new knowledge was identified and solutions to some missing is-a relations were refined. Therefore, our approach for completing the is-a structures can be seen as a detection method where the input is a set of is-a relations that require inspection, and the output is new knowledge to be added to the ontology.

With respect to alignments, we have conducted a case study of the last 10 years of the anatomy track at Ontology Alignment Evaluation Initiative, which is one of the longest running tracks of the campaign. We studied system performance and also looked at the approaches used by the systems to get a better understanding of the state of the art and identify possible shortcomings and directions for improvement. Our analysis has shown that runtimes of the participating systems have improved significantly over the years. However, while the precision of the participating systems is usually very high, many of the systems fail in finding non-trivial correspondences, i.e. correspondences which cannot be found by simple string matching. For example, even after ten years of the track there are correspondences that were found by only a few systems.

Furthermore, we have also addressed the problem of scalability, which is one of the challenges facing the community. We have developed a method for reducing the search space for matching algorithms that is based on clustering and text mining. Clustering is used to partition the ontologies into smaller partitions. Text mining methods are then used to identify topics in the partitions. Therefore, the matching algorithms need only to compare 
concepts in clusters with similar topics in two ontologies. Our evaluation has shown that our method cuts the search space significantly, thus reducing the effort for computation as well as validation while still maintaining high precision. However, other techniques will be needed to deal with the low recall, given that some concepts end up being outliers in the clustering phase.

User involvement is necessary to ensure high-quality completion of ontologies and ontology networks. We conducted a broad study which identified three aspects of user validation in ontology alignment, the user's profile, ontology alignment system services and their user interfaces. The services and the user interface were assessed in a qualitative evaluation of the stateof-the-art systems while the impact of user errors, as well as how systems cope with them, were evaluated in experiments where user interaction was simulated using an oracle.

The user profile is one of the key factors in user validation. With respect to the user profile, we have identified three different types of expertise: domain expertise of the user, technical expertise, and expertise with the alignment systems. These set the requirements on how the user interface should be implemented, which information can be shown, and the degree of complexity that can be included. To facilitate the validation process for the user, the user interface should provide components such as explanations of the correspondences, contextual information about the correspondences, the consequences of validating a correspondence, and should also provide mechanisms for annotating correspondences or enabling validation over multiple sessions. However, in all cases systems should not overwhelm the user with information, as users have limited working memory and limited availability. To deal with limited availability, systems can provide system services for prioritizing the correspondence suggestions that are presented to the user, and then extrapolate the user's input to similar correspondences (using methods such as feedback propagation). However, our experiments have shown that extrapolation can increase the impact of user errors, as erroneous user input will be extended to other correspondence suggestions. Therefore, systems need to find a balance in the number of suggestions passed to the user and the degree of extrapolation.

Completion of ontologies and ontology networks can be done at any point in their life-cycle, however some of the issues can be addressed early, in the development phase. Our work presents an approach for integrating ontology debugging, completion and alignment into the eXtreme Design Methodology (XD), which is an ontology development methodology based on reusing ontology patterns, i.e. small ontology fragments which represent general use cases. To evaluate the approach we applied it to an existing ontology which was developed using the XD methodology. Our analysis has found a number of issues with the developed ontology which could have been addressed in the development phase using our approach. 


\section{Future work}

There are a number of directions that are interesting for future work. They focus on improvements to the current approaches as well as extensions such as dealing with more expressive languages, and different methodologies. Here we list some of the interesting directions for future work:

\section{Completing the is-a structure}

Our approach for repairing missing is-a relations can be regarded as a detection method given that solutions to GTAP often contain new is-a relations which were not detected by the detection algorithm. However, these detection mechanisms explore only small parts of ontologies that are related to is-a relations under inspection. Therefore, a detection component would be beneficial for our approach as it would identify additional missing is-a relations and provide a starting point for the process of completing ontologies. There are many approaches for detecting missing is-a relations in ontologies, some of which were described in Chapter 4.

Furthermore, it is necessary to evaluate the scalability of our approach to more expressive representation languages as well as different types of relations. Some scalability issues could be alleviated by considering modular approaches where algorithms are run only on the parts of ontologies that are relevant for a certain missing relation.

\section{User validation in the completion process}

Our work analysing user validation has identified three different types of user expertise. Users can have different types and different levels of expertise. In order to evaluate the interplay between different aspects of the system (user interface and system services) and their impact on different types and levels of user's expertise, it would be beneficial to conduct usability testing, which would provide a better understanding of the requirements for ontology matching systems for users with different expertise types and levels.

In addition, another interesting direction related to user validation is to evaluate different validation approaches, such as presenting a user with conflicting mappings, sets of similar mappings, or giving the user the ability to assign a confidence value instead of making a binary decision.

\section{Partitioning the search space}

In our approach for partitioning the search space in ontology matching after the clustering step it is necessary to find representative keywords in each cluster, which are then used to identify topics for each cluster. In the current implementation, topic identification, as well as mapping of clusters with similar topics, is done manually. Therefore, one direc- 
tion for future work would be to look at approaches for automating this step.

Another future work direction would be to extend the current approach to increase recall. In this case we would have to consider outliers, which in the current approach are excluded from the partitions.

\section{Quality assurance in ontology development methodologies}

The proposed approach requires a set of different tools which need to be run on the target ontology in order to integrate the ontology patterns and repair different defects. Therefore, one direction for future work would be to develop a support tool for ontology development which would include the various steps discussed in our approach.

In addition, our approach for integrating ontology matching and ontology debugging into ontology development was applied on one methodology. Therefore, a more general method for quality assurance in ontology development is needed. This would also require conducting usability studies to evaluate the added value for users. 



\section{Bibliography}

[1] BioPortal. http://bioportal.bioontology.org/. Accessed: 201706-08.

[2] Internet Live Stats. http://www.internetlivestats.com/. Accessed: 2017-05-13.

[3] MeSH: Medical Subject Headings. http://www.nlm.nih.gov/mesh/. Accessed: 2017-06-08.

[4] Netcraft Web Server Survey. https://news.netcraft.com/ archives/2017/05/25/may-2017-web-server-survey.html. Accessed: 2017-05-13.

[5] PubMed. http://www.ncbi.nlm.nih.gov/pubmed. Accessed: 201706-08.

[6] SNOMED Clinical Terms. http://www.ihtsdo.org/snomed-ct/. Accessed: 2017-6-08.

[7] UMLS Metathesaurus: Unified Medical Language System Metathesaurus. http://www.nlm.nih.gov/research/umls/knowledge_ sources/metathesaurus/. Accessed: 2017-06-08.

[8] WordNet. http://wordnet.princeton.edu/. Accessed: 2017-06-08.

[9] J. H. Alexander, M. J. Freiling, S. Shulman, J. Staley, S. Rehfuss, and S. Messick. Knowledge level engineering ontological analysis. In Proceedings of the 5th National Conference on Artificial Intelligence (AAAI), pages 963-968, 1986.

[10] P. Arnold and E. Rahm. Semantic enrichment of ontology mappings: a linguistic-based approach. In Proceedings of the 17th East-European Conference on Advances in Databases and Information Systems (AD$B I S)$, pages $42-55,2013$.

[11] F. Baader and W. Nutt. Basic description logics. In F. Baader, D. Calvanese, D. McGuinness, D. Nardi, and P. Patel-Schneider, editors, Description logic handbook, chapter 2, pages 43-95. 2003. 
[12] F. Baader and U. Sattler. An overview of tableau algorithms for description logics. Studia Logica, 69:5-40, 2001.

[13] M. Bada and L. Hunter. Identification of OBO nonalignments and its implications for OBO enrichment. Bioinformatics, 24(12):1448-1455, 2008.

[14] Z. Bellahsene, A. Bonifati, and E. Rahm. Schema matching and mapping. Springer, 2011.

[15] T. Berners-Lee, J. Hendler, and O. Lassila. The Semantic Web. Scientific American, 284(5):28-37, 2001.

[16] M. Bienvenu. Complexity of abduction in the $\mathcal{E} \mathcal{L}$ family of lightweight description logics. In Proceedings of the 11th International Conference on Principles of Knowledge Representation and Reasoning (KR), pages 220-230, 2008.

[17] D. M. Blei, A. Y. Ng, and M. I. Jordan. Latent Dirichlet Allocation. Journal of Machine Learning Research, 3:993-1022, 2003.

[18] O. Bodenreider, T. F. Hayamizu, M. Ringwald, S. De Coronado, and S. Zhang. Of mice and men: Aligning mouse and human anatomies. In Proceedings of the 2005 AMIA Annual Symposium, pages 61-65, 2005.

[19] A. Borgida and P. Patel-Schneider. A semantics and complete algorithm for subsumption in the CLASSIC description logic. Journal of Artificial Intelligence Research, 1:277-308, 1994.

[20] P. Buitelaar, P. Cimiano, and B. Magnini. Ontology Learning from Text: Methods, Evaluation and Applications. IOS Press, 2005.

[21] A. Chiatti, Z. Dragisic, T. Cerquitelli, and P. Lambrix. Reducing the search space in ontology alignment using clustering techniques and topic identification. In Proceedings of the 8th International Conference on Knowledge Capture (K-CAP), number 21, 2015.

[22] P. Cimiano, A. Hotho, and S. Staab. Learning concept hierarchies from text corpora using formal concept analysis. Journal of Artifcial Intelligence Research, 24:305-339, 2005.

[23] S. Colucci, T. Di Noia, E. Di Sciascio, F. Donini, and M. Mongiello. A uniform tableaux-based approach to concept abduction and contraction in $\mathcal{A L N}$. In Proceedings of the 17th International Workshop on Description Logics (DL), pages 158-167, 2004.

[24] O. Corcho, M. Fernández-López, and A. Gómez-Pérez. Ontological engineering: principles, methods, tools and languages. In C. Calero, F. Ruiz, and M. Piattini, editors, Ontologies for software engineering and software technology, chapter 1, pages 1-48. 2006. 
[25] O. Corcho, C. Roussey, L. M. Vilches, and I. Pérez. Pattern-based OWL ontology debugging guidelines. In Proceedings of the 1st Workshop on Ontology Patterns (WOP), pages 68-82, 2009.

[26] B. Cuenca Grau, I. Horrocks, Y. Kazakov, and U. Sattler. Just the right amount: extracting modules from ontologies. In Proceedings of the 16th International Conference on World Wide Web (WWW), pages 717-726, 2007.

[27] J. C. de Almeida Biolchini, P. G. Mian, A. C. C. Natali, T. U. Conte, and G. H. Travassos. Scientific research ontology to support systematic review in software engineering. Advanced Engineering Informatics, 21(2):133-151, 2007.

[28] T. Di Noia, E. Di Sciascio, and F. Donini. Semantic matchmaking as non-monotonic reasoning: A description logic approach. Journal of Artificial Intelligence Research, 29:269-307, 2007.

[29] H. Do and E. Rahm. Matching large schemas: Approaches and evaluation. Information Systems, 32(6):857-885, 2007.

[30] F. Donini, S. Colucci, T. Di Noia, and E. Di Sciasco. A tableaux-based method for computing least common subsumers for expressive description logics. In Proceedings of the 21st International Joint Conference on Artificial Intelligence (IJCAI), pages 739-745, 2009.

[31] Z. Dragisic, V. Ivanova, P. Lambrix, D. Faria, E. Jiménez-Ruiz, and C. Pesquita. User validation in ontology alignment. In Proceedings of the 15th International Semantic Web Conference (ISWC), pages 200-217, 2016.

[32] Z. Dragisic, V. Ivanova, H. Li, and P. Lambrix. Experiences from the anatomy track in the Ontology Alignment Evaluation Initiative. submitted.

[33] Z. Dragisic, P. Lambrix, and E. Blomqvist. Integrating ontology debugging and matching into the eXtreme Design methodology. In Proceedings of the 6th Workshop on Ontology and Semantic Web Patterns (WOP), 2015.

[34] J. Du, G. Qi, Y. Shen, and J. Pan. Towards practical Abox abduction in large OWL DL ontologies. In Proceedings of the 25th AAAI Conference on Artificial Intelligence, pages 1160-1165, 2011.

[35] J. Du, G. Qi, Y. Shen, and J. Pan. Towards practical ABox abduction in large description logic ontologies. International Journal on Semantic Web and Information Systems (IJSWIS), 8(2):1-33, 2012. 
[36] J. Du, H. Wan, and H. Ma. Practical TBox abduction based on justification patterns. In Proceedings of the 31st AAAI Conference on Artificial Intelligence, pages 1100-1106, 2017.

[37] J. Du, K. Wang, and Y. Shen. A tractable approach to ABox abduction over description logic ontologies. In Proceedings of the 28th AAAI Conference on Artificial Intelligence, pages 1034-1040, 2014.

[38] K. M. Eisenhardt. Building theories from case study research. Academy of management review, 14(4):532-550, 1989.

[39] T. Eiter and G. Gottlob. The complexity of logic-based abduction. Journal of the ACM, 42(1):3-42, 1995.

[40] C. Elsenbroich, O. Kutz, and U. Sattler. A case for abductive reasoning over ontologies. In Proceedings of the 2nd International Workshop OWL: Experiences and Directions (OWLED), 2006.

[41] J. Euzenat and P. Shvaiko. Ontology matching. Springer, 2007.

[42] J. Euzenat and P. Shvaiko. User involvement. In J. Euzenat and P. Shvaiko, editors, Ontology Matching, chapter 11, pages 353-375. Springer, 2013.

[43] S. Falconer and N. Noy. Interactive techniques to support ontology matching. In Z. Bellahsene, A. Bonifati, and E. Rahm, editors, Schema Matching and Mapping, chapter 2, pages 29-51. Springer, 2011.

[44] S. Falconer and M. Storey. A cognitive support framework for ontology mapping. In Proceedings of the 6th International Semantic Web Conference (ISWC) and the 2nd Asian Semantic Web Conference $(A S W C)$, pages 114-127, 2007.

[45] G. Flouris, D. Manakanatas, H. Kondylakis, D. Plexousakis, and G. Antoniou. Ontology Change: Classification and Survey. Knowledge Engineering Review, 23(2):117-152, 2008.

[46] B. Ganter and R. Wille. Formal concept analysis. Springer, 1999.

[47] M. Granitzer, V. Sabol, K. W. Onn, D. Lukose, and K. Tochtermann. Ontology alignment - a survey with focus on visually supported semiautomatic techniques. Future Internet, 2(3):238-258, 2010.

[48] A. Groß, M. Hartung, T. Kirsten, and E. Rahm. GOMMA results for OAEI 2012. In Proceedings of the 7th International Workshop on Ontology Matching (OM), pages 133-140, 2012.

[49] T. R. Gruber. A translation approach to portable ontology specifications. Knowledge acquisition, 5(2):199-220, 1993. 
[50] N. Guarino. Some ontological principles for designing upper level lexical resources. In Proceedings of the 1st International Conference on Language Resources and Evaluation (LREC), pages 527-534, 1998.

[51] S. Guha, R. Rastogi, and K. Shim. ROCK: A robust clustering algorithm for categorical attributes. Information systems, 25(5):345-366, 2000 .

[52] P. Haase and Lj. Stojanovic. Consistent Evolution of OWL Ontologies. In Proceedings of the 2nd European Semantic Web Conference (ESWC), pages 182-197, 2005.

[53] K. Halland and K. Britz. Naive ABox abduction in $\mathcal{A L C}$ using a DL tableau. In Proceedings of the 25th International Workshop on Description Logics (DL), pages 443-453, 2012.

[54] K. Halland, K. Britz, and S. Klarman. TBox abduction in $\mathcal{A L C}$ using a DL tableau. In Proceedings of the 27th International Workshop on Description Logics (DL), pages 556-566, 2014.

[55] H. J. Happel and S. Seedorf. Applications of ontologies in software engineering. In Proceedings of the 2nd Workshop on Semantic Web Enabled Software Engineering Workshop on Sematic Web Enabled Software Engineering (SWESE), pages 5-9, 2006.

[56] M. Hartung, J. Terwilliger, and E. Rahm. Recent advances in schema and ontology evolution. In Z. Bellahsene, A. Bonifati, and E. Rahm, editors, Schema matching and mapping, chapter 6, pages 149-190. 2011.

[57] M. Hearst. Automatic acquisition of hyponyms from large text corpora. In Proceedings of the 14th International Conference on Computational Linguistics (COLING), pages 539-545, 1992.

[58] A. Herzog, N. Shahmehri, and C. Duma. An ontology of information security. International Journal of Information Security and Privacy (IJISP), 1(4):1-23, 2007.

[59] W. Hu, Y. Qu, and G. Cheng. Matching large ontologies: A divideand-conquer approach. Data \& Knowledge Engineering, 67(1):140$160,2008$.

[60] T. Hubauer, S. Lamparter, and M. Pirker. Automata-based abduction for tractable diagnosis. In Proceedings of the 23rd International Workshop on Description Logics (DL), pages 360-371, 2010.

[61] V. Ivanova and P. Lambrix. A unified approach for aligning taxonomies and debugging taxonomies and their alignments. In Proceedings of the 10th Extended Semantic Web Conference (ESWC), pages 1-15, 2013. 
[62] V. Ivanova, P. Lambrix, and J. Åberg. Requirements for and evaluation of user support for large-scale ontology alignment. In Proceedings of the 15th European Semantic Web Conference (ESWC), pages 3-20, 2015.

[63] V. Ivanova, J. Laurila Bergman, U. Hammerling, and P. Lambrix. Debugging taxonomies and their alignments: the ToxOntology - MeSH use case. In Proceedings of the 1st International Workshop on Debugging Ontologies and Ontology Mappings (WoDOOM), pages 25-36, 2012 .

[64] Q. Ji, P. Haase, G. Qi, P. Hitzler, and S. Stadtmuller. RaDON - repair and diagnosis in ontology networks. In Proceedings of the 6th European Semantic Web Conference (ESWC), pages 863-867, 2009.

[65] Y. Kalfoglou and M. Schorlemmer. Ontology mapping: the state of the art. The knowledge engineering review, 18(01):1-31, 2003.

[66] A. Kalyanpur. Debugging and repair of OWL ontologies. PhD thesis, University of Maryland, 2006.

[67] A. Kalyanpur, B. Parsia, M. Horridge, and E. Sirin. Finding all justifications of OWL DL entailments. In Proceedings of the 6th International Semantic Web Conference (ISWC) and the 2nd Asian Semantic Web Conference (ASWC), pages 267-280. 2007.

[68] A. Kalyanpur, B. Parsia, E. Sirin, and B. Cuenca Grau. Repairing unsatisfiable concepts in OWL ontologies. In Proceedings of the 3rd European Semantic Web Conference (ESWC), pages 170-184, 2006.

[69] A. Kalyanpur, B. Parsia, E. Sirin, and J. Hendler. Debugging Unsatisfiable Classes in OWL Ontologies. Journal of Web Semantics, 3(4):268-293, 2006.

[70] C. M. Keet. Detecting and revising flaws in OWL object property expressions. In Proceedings of the 18th Conference on Knowledge Engineering and Knowledge Management (EKAW), pages 252-266, 2012.

[71] A. Kim, J. Luo, and M. Kang. Security ontology for annotating resources. In Proceedings of the 4th International Conference on Ontologies, Databases, and Applications of Semantics (ODBASE), pages 1483-1499. 2005.

[72] B. Kitchenham. Procedures for performing systematic reviews. Technical report - Keele University, 33(2004):1-26, 2004.

[73] S. Klarman, U. Endriss, and S. Schlobach. ABox abduction in the description logic $\mathcal{A L C}$. Journal of Automated Reasoning, 46:43-80, 2011. 
[74] P. Koopmann and R. A. Schmidt. LETHE: Saturation-based reasoning for non-standard reasoning tasks. In Proceedings of the 4th $O W L$ Reasoning Evaluation Workshop (ORE), pages 23-30, 2015.

[75] P. Lambrix. Ontologies in bioinformatics and systems biology. In W. Dubitzky and F. Azuaje, editors, Artificial Intelligence Methods and Tools for Systems Biology, chapter 8, pages 129-145. 2004.

[76] P. Lambrix. Towards a semantic web for bioinformatics using ontologybased annotation. In Proceedings of 14 th IEEE International Workshops on the Enabling Technologies: Infrastructure for Collaborative Enterprise, pages 3-7, 2005.

[77] P. Lambrix, Z. Dragisic, and V. Ivanova. Get my pizza right: Repairing missing is-a relations in $\mathcal{A L C}$ ontologies. In Proceedings of the 2nd Joint International Semantic Technology Conference (JIST), pages $17-32,2012$.

[78] P. Lambrix and A. Edberg. Evaluation of ontology merging tools in bioinformatics. In Proceedings of the Pacific Symposium on Biocomputing, volume 8, pages 589-600, 2003.

[79] P. Lambrix and V. Ivanova. A unified approach for debugging is-a structure and mappings in networked taxonomies. Journal of Biomedical Semantics, 4:10:1-10:19, 2013.

[80] P. Lambrix and R. Kaliyaperumal. A session-based ontology alignment approach enabling user involvement. Semantic Web Journal, 8(2):225$251,2017$.

[81] P. Lambrix and Q. Liu. Using partial reference alignments to align ontologies. In Proceedings of the 6th European Semantic Web Conference (ESWC), pages 188-202, 2009.

[82] P. Lambrix and Q. Liu. Debugging is-a structure in networked taxonomies. In Proceedings of the 4th International Workshop on Semantic Web Applications and Tools for Life Sciences (SWAT4LS), pages 58-65, 2011.

[83] P. Lambrix and Q. Liu. Debugging the missing is-a structure within taxonomies networked by partial reference alignments. Data $\&$ Knowledge Engineering, 86:179-205, 2013.

[84] P. Lambrix, Q. Liu, and H. Tan. Repairing the missing is-a structure of ontologies. In Proceedings of the 4th Asian Semantic Web Conference (ASWC), pages 76-90, 2009.

[85] P. Lambrix, L. Strömbäck, and H. Tan. Information integration in bioinformatics with ontologies and standards. In F. Bry and 
J. Maluszynski, editors, Semantic Techniques for the Web: The REWERSE perspective, chapter 8, pages 343-376. 2009.

[86] P. Lambrix and H. Tan. SAMBO - a system for aligning and merging biomedical ontologies. Journal of Web Semantics, 4(3):196-206, 2006.

[87] P. Lambrix, F. Wei-Kleiner, and Z. Dragisic. Completing the is-a structure in light-weight ontologies. Journal of Biomedical Semantics, $6(1): 12,2015$.

[88] O. Lassila and D. McGuinness. The role of frame-based representation on the Semantic Web. Technical report, 2001.

[89] D. B. Lenat. CYC: A large-scale investment in knowledge infrastructure. Communications of the ACM, 38(11):33-38, 1995.

[90] C. Lutz. Complexity of terminiological reasoning revisited. In Proceedings of the 16th International Conference on Logic for Programming and Automated Reasoning (LPAR), pages 181-200, 1999.

[91] A. Maedche and S. Staab. Discovering conceptual relations from text. In Proceedings of the 14th European Conference on Artificial Intelligence (ECAI), pages 321-325, 2000.

[92] J. McCarthy. Circumscription - a form of non-monotonic reasoning. Artificial Intelligence, 13(1):27-39, 1980.

[93] C. Meilicke, H. Stuckenschmidt, and A. Tamilin. Repairing Ontology Mappings. In Proceedings of the 22nd AAAI Conference on Artificial Intelligence, pages 1408-1413, 2007.

[94] R. Neches, R. E. Fikes, T. Finin, T. Gruber, R. Patil, T. Senator, and W. R. Swartout. Enabling technology for knowledge sharing. AI magazine, 12(3):36, 1991.

[95] N. Noy. Semantic integration: a survey of ontology-based approaches. ACM Sigmod Record, 33(4):65-70, 2004.

[96] N. Noy, N. H. Shah, P. L. Whetzel, B. Dai, M. Dorf, N. Griffith, C. Jonquet, D. L. Rubin, M. Storey, C. G. Chute, and M. A. Musen. Bioportal: ontologies and integrated data resources at the click of a mouse. Nucleic acids research, 37(suppl 2):W170-W173, 2009.

[97] L. Otero-Cerdeira, F. J. Rodríguez-Martínez, and A. GómezRodríguez. Ontology matching: A literature review. Expert Systems with Applications, 42(2):949-971, 2015.

[98] H. Paulheim. On applying matching tools to large-scale ontologies. In Proceedings of the 3rd International Workshop on Ontology Matching (OM), pages 214-218, 2008. 
[99] M. Poveda-Villalón, M. C. Suárez-Figueroa, and A. Gómez-Pérez. Validating ontologies with oops! In Proceedings of the 18th International Conference on Knowledge Engineering and Knowledge Management (EKAW), pages 267-281, 2012.

[100] G. Qi, Q. Ji, and P. Haase. A conflict-based operator for mapping revision. In Proceedings of the 8th International Semantic Web Conference (ISWC), pages 521-536, 2009.

[101] J. C. Dos Reis, D. Dinh, C. Pruski, M. Da Silveira, and C. ReynaudDelaître. Mapping adaptation actions for the automatic reconciliation of dynamic ontologies. In Proceedings of the 22nd ACM International Conference on Information \& Knowledge Management (CIKM), pages 599-608, 2013.

[102] R. Reiter. A theory of diagnosis from first principles. Artificial Intelligence, 32(1):57-95, 1987.

[103] C. Roussey and A. Zamazal. Antipattern detection: How to debug an ontology without a reasoner. In Proceedings of the 2nd International Workshop on Debugging Ontologies and Ontology Mappings (WoDOOM), pages 45-56, 2013.

[104] S. Schlobach. Debugging and Semantic Clarification by Pinpointing. In Proceedings of the 2nd European Semantic Web Conference (ESWC), pages 226-240, 2005.

[105] S. Schlobach and R. Cornet. Non-standard reasoning services for the debugging of description logic terminologies. In Proceedings of the 18th International Joint Conferences on Artificial Intelligence (IJCAI), volume 3, pages 355-362, 2003.

[106] M. Schmidt-Schauß and G. Smolka. Attributive concept descriptions with complements. Artificial Intelligence, 48(1):1-26, 1991.

[107] M. H. Seddiqui and M. Aono. An efficient and scalable algorithm for segmented alignment of ontologies of arbitrary size. Web Semantics: Science, Services and Agents on the World Wide Web, 7(4):344-356, 2009.

[108] K. Shchekotykhin, G. Friedrich, P. Fleiss, and P. Rodler. Interactive ontology debugging: Two query strategies for efficient fault localization. Journal of Web Semantics, 12:88-103, 2012.

[109] P. Shvaiko and J. Euzenat. A survey of schema-based matching approaches. In Journal on Data Semantics, volume IV, pages 146-171. Springer, 2005. 
[110] P. Shvaiko and J. Euzenat. Ten challenges for ontology matching. On the Move to Meaningful Internet Systems: OTM 2008, pages 1164$1182,2008$.

[111] P. Shvaiko and J. Euzenat. Ontology matching: state of the art and future challenges. IEEE Transactions on knowledge and data engineering, 25(1):158-176, 2013.

[112] E. Simperl, M. Mochol, and T. Bürger. Achieving maturity: The state of practice in ontology engineering in 2009. Int Journal of Computer Science and Applications, 7(1):45-65, 2010.

[113] V. Spiliopoulos, G. A. Vouros, and V. Karkaletsis. On the discovery of subsumption relations for the alignment of ontologies. Web Semantics: Science, Services and Agents on the World Wide Web, 8(1):69-88, 2010.

[114] R. Stevens, C. A. Goble, and S. Bechhofer. Ontology-based knowledge representation for bioinformatics. Briefings in Bioinformatics, 1(4):398-414, 2000.

[115] H. Stuckenschmidt and M. Klein. Structure-based partitioning of large concept hierarchies. In Proceedings of the 3rd International Semantic Web Conference (ISWC), pages 289-303, 2004.

[116] R. Studer, R. V. Benjamins, and D. Fensel. Knowledge engineering: principles and methods. Data \& knowledge engineering, 25(1):161$197,1998$.

[117] M. Uschold and M. Gruninger. Ontologies and semantics for seamless connectivity. ACM SIGMod Record, 33(4):58-64, 2004.

[118] T. Wächter, H.Tan, A. Wobst, P. Lambrix, and M. Schroeder. A corpus-driven approach for design, evolution and alignment of ontologies. In Proceedings of the 38th Winter Simulation Conference, pages 1595-1602, 2006.

[119] P. Wang and B. Xu. Debugging ontology mappings: a static approach. Computing and Informatics, 27:21-36, 2008.

[120] P. Wang, Y. Zhou, and B. Xu. Matching large ontologies based on reduction anchors. In Proceedings of the 22nd International Joint Conference on Artificial Intelligence (IJCAI), pages 2343-2348, 2011.

[121] F. Wei-Kleiner, Z. Dragisic, and P. Lambrix. Abduction framework for repairing incomplete $\mathcal{E} \mathcal{L}$ ontologies: Complexity results and algorithms. In Proceedings of the 28th AAAI Conference on Artificial Intelligence, pages 1120-1127, 2014.

[122] C. Welty. Ontology research. AI magazine, 24(3):11, 2003. 
[123] E. Zavitsanos, G. Paliouras, G. A. Vouros, and S. Petridis. Discovering subsumption hierarchies of ontology concepts from text corpora. In Proceedings of the 2007 IEEE/WIC/ACM International Conference on Web Intelligence, pages 402-408, 2007. 


\section{Papers}

The articles associated with this thesis have been removed for copyright reasons. For more details about these see:

http://urn.kb.se/resolve? urn:nbn:se:liu:diva-139487 


\section{Dissertations}

\section{Linköping Studies in Science and Technology Linköping Studies in Arts and Science}

Linköping Studies in Statistics

Linköping Studies in Information Science

\section{Linköping Studies in Science and Technology}

No 14 Anders Haraldsson: A Program Manipulation System Based on Partial Evaluation, 1977, ISBN 917372-144-1.

No 17 Bengt Magnhagen: Probability Based Verification of Time Margins in Digital Designs, 1977, ISBN 91-7372157-3.

No 18 Mats Cedwall: Semantisk analys av processbeskrivningar i naturligt språk, 1977, ISBN 91- 7372168-9.

No 22 Jaak Urmi: A Machine Independent LISP Compiler and its Implications for Ideal Hardware, 1978, ISBN 91-7372-188-3.

No 33 Tore Risch: Compilation of Multiple File Queries in a Meta-Database System 1978, ISBN 91-7372-232-4.

No 51 Erland Jungert: Synthesizing Database Structures from a User Oriented Data Model, 1980, ISBN 917372-387-8.

No 54 Sture Hägglund: Contributions to the Development of Methods and Tools for Interactive Design of Applications Software, 1980, ISBN 91-7372-404-1.

No 55 Pär Emanuelson: Performance Enhancement in a Well-Structured Pattern Matcher through Partial Evaluation, 1980, ISBN 91-7372-403-3.

No 58 Bengt Johnsson, Bertil Andersson: The HumanComputer Interface in Commercial Systems, 1981, ISBN 91-7372-414-9.

No 69 H. Jan Komorowski: A Specification of an Abstract Prolog Machine and its Application to Partial Evaluation, 1981, ISBN 91-7372-479-3.

No 71 René Reboh: Knowledge Engineering Techniques and Tools for Expert Systems, 1981, ISBN 91-7372489-0.

No 77 Östen Oskarsson: Mechanisms of Modifiability in large Softw are Systems, 1982, ISBN 91- 7372-527-7.

No 94 Hans Lunell: Code Generator Writing Systems, 1983, ISBN 91-7372-652-4.

No 97 Andrzej Lingas: Advances in Minimum Weight Triangulation, 1983, ISBN 91-7372-660-5.

No 109 Peter Fritzson: Towards a Distributed Programming Environment based on Incremental Compilation, 1984, ISBN 91-7372-801-2.

No 111 Erik Tengvald: The Design of Expert Planning Systems. An Experimental Operations Planning System for Turning, 1984, ISBN 91-7372- 805-5.

No 155 Christos Levcopoulos: Heuristics for Minimum Decompositions of Polygons, 1987, ISBN 91-7870133-3.

No 165 James W. Goodwin: A Theory and System for NonMonotonic Reasoning, 1987, ISBN 91-7870-183-X.

No 170 Zebo Peng: A Formal Methodology for Automated Synthesis of VLSI Systems, 1987, ISBN 91-7870-225-9.

No 174 Johan Fagerström: A Paradigm and System for Design of Distributed Systems, 1988, ISBN 91-7870301-8.

No 192 Dimiter Driankov: Towards a Many Valued Logic of Quantified Belief, 1988, ISBN 91-7870-374-3.
No 213 Lin Padgham: Non-Monotonic Inheritance for an Object Oriented Knowledge Base, 1989, ISBN 917870-485-5.

No 214 Tony Larsson: A Formal Hardware Description and Verification Method, 1989, ISBN 91-7870-517-7.

No 221 Michael Reinfrank: Fundamentals and Logical Foundations of Truth Maintenance, 1989, ISBN 917870-546-0.

No 239 Jonas Löwgren: Knowledge-Based Design Support and Discourse Management in User Interface Management Systems, 1991, ISBN 91-7870-720-X.

No 244 Henrik Eriksson: Meta-Tool Support for Knowledge Acquisition, 1991, ISBN 91-7870-746-3.

No 252 Peter Eklund: An Epistemic Approach to Interactive Design in Multiple Inheritance Hierarchies, 1991, ISBN 91-7870-784-6.

No 258 Patrick Doherty: NML3 - A Non-Monotonic Formalism with Explicit Defaults, 1991, ISBN 917870-816-8.

No 260 Nahid Shahmehri: Generalized Algorithmic Debugging, 1991, ISBN 91-7870-828-1.

No 264 Nils Dahlbäck: Representation of DiscourseCognitive and Computational Aspects, 1992, ISBN 91-7870-850-8.

No 265 Ulf Nilsson: Abstract Interpretations and Abstract Machines: Contributions to a Methodology for the Implementation of Logic Programs, 1992, ISBN 917870-858-3.

No 270 Ralph Rönnquist: Theory and Practice of Tensebound Object References, 1992, ISBN 91-7870-873-7.

No 273 Björn Fjellborg: Pipeline Extraction for VLSI Data Path Synthesis, 1992, ISBN 91-7870-880-X.

No 276 Staffan Bonnier: A Formal Basis for Horn Clause Logic with External Polymorphic Functions, 1992, ISBN 91-7870-896-6.

No 277 Kristian Sandahl: Developing Knowledge Management Systems with an Active Expert Methodology, 1992, ISBN 91-7870-897-4.

No 281 Christer Bäckström: Computational Complexity of Reasoning about Plans, 1992, ISBN 91-7870-979-2.

No 292 Mats Wirén: Studies in Incremental Natural Language Analysis, 1992, ISBN 91-7871-027-8.

No 297 Mariam Kamkar: Interprocedural Dynamic Slicing with Applications to Debugging and Testing, 1993, ISBN 91-7871-065-0.

No 302 Tingting Zhang: A Study in Diagnosis Using Classification and Defaults, 1993, ISBN 91-7871-078-2

No 312 Arne Jönsson: Dialogue Management for Natural Language Interfaces - An Empirical Approach, 1993, ISBN 91-7871-110-X

No 338 Simin Nadjm-Tehrani: Reactive Systems in Physical Environments: Compositional Modelling and Framework for Verification, 1994, ISBN 91-7871-237-8.

No 371 Bengt Savén: Business Models for Decision Support and Learning. A Study of Discrete-Event Manufacturing Simulation at Asea/ ABB 1968-1993, 1995, ISBN 91-7871-494-X. 
No 375 Ulf Söderman: Conceptual Modelling of Mode Switching Physical Systems, 1995, ISBN 91-7871-5164.

No 383 Andreas Kågedal: Exploiting Groundness in Logic Programs, 1995, ISBN 91-7871-538-5.

No 396 George Fodor: Ontological Control, Description, Identification and Recovery from Problematic Control Situations, 1995, ISBN 91-7871-603-9.

No 413 Mikael Pettersson: Compiling Natural Semantics, 1995, ISBN 91-7871-641-1.

No 414 Xinli Gu: RT Level Testability Improvement by Testability Analysis and Transformations, 1996, ISBN 91-7871-654-3.

No 416 Hua Shu: Distributed Default Reasoning, 1996, ISBN 91-7871-665-9.

No 429 Jaime Villegas: Simulation Supported Industrial Training from an Organisational Learning Perspective - Development and Evaluation of the SSIT Method, 1996, ISBN 91-7871-700-0.

No 431 Peter Jonsson: Studies in Action Planning: Algorithms and Complexity, 1996, ISBN 91-7871-7043.

No 437 Johan Boye: Directional Types in Logic Programming, 1996, ISBN 91-7871-725-6.

No 439 Cecilia Sjöberg: Activities, Voices and Arenas: Participatory Design in Practice, 1996, ISBN 91-7871728-0.

No 448 Patrick Lambrix: Part-Whole Reasoning in Description Logics, 1996, ISBN 91-7871-820-1.

No 452 Kjell Orsborn: On Extensible and Object-Relational Database Technology for Finite Element Analysis Applications, 1996, ISBN 91-7871-827-9.

No 459 Olof Johansson: Development Environments for Complex Product Models, 1996, ISBN 91-7871-855-4.

No 461 Lena Strömbäck: User-Defined Constructions in Unification-Based Formalisms, 1997, ISBN 91-7871857-0.

No 462 Lars Degerstedt: Tabulation-based Logic Programming: A Multi-Level View of Query Answering, 1996, ISBN 91-7871-858-9.

No 475 Fredrik Nilsson: Strategi och ekonomisk styrning En studie av hur ekonomiska styrsystem utformas och används efter företagsförvärv, 1997, ISBN 917871-914-3.

No 480 Mikael Lindvall: An Empirical Study of Requirements-Driven Impact Analysis in Object-Oriented Software Evolution, 1997, ISBN 91-7871-927-5.

No 485 Göran Forslund: Opinion-Based Systems: The Cooperative Perspective on Knowledge-Based Decision Support, 1997, ISBN 91-7871-938-0.

No 494 Martin Sköld: Active Database Management Systems for Monitoring and Control, 1997, ISBN 917219-002-7.

No 495 Hans Olsén: Automatic Verification of Petri Nets in a CLP framework, 1997, ISBN 91-7219-011-6.

No 498 Thomas Drakengren: Algorithms and Complexity for Temporal and Spatial Formalisms, 1997, ISBN 917219-019-1.

No 502 Jakob Axelsson: Analysis and Synthesis of Heterogeneous Real-Time Systems, 1997, ISBN 91-7219-035-3.

No 503 Johan Ringström: Compiler Generation for DataParallel Programming Languages from Two-Level Semantics Specifications, 1997, ISBN 91-7219-045-0.

No 512 Anna Moberg: Närhet och distans - Studier av kommunikationsmönster i satellitkontor och flexibla kontor, 1997, ISBN 91-7219-119-8.
No 520 Mikael Ronström: Design and Modelling of a Parallel Data Server for Telecom Applications, 1998, ISBN 91-7219-169-4.

No 522 Niclas Ohlsson: Towards Effective Fault Prevention - An Empirical Study in Software Engineering, 1998, ISBN 91-7219-176-7.

No 526 Joachim Karlsson: A Systematic Approach for Prioritizing Software Requirements, 1998, ISBN 917219-184-8.

No 530 Henrik Nilsson: Declarative Debugging for Lazy Functional Languages, 1998, ISBN 91-7219-197-x.

No 555 Jonas Hallberg: Timing Issues in High-Level Synthesis, 1998, ISBN 91-7219-369-7.

No 561 Ling Lin: Management of 1-D Sequence Data - From Discrete to Continuous, 1999, ISBN 91-7219-402-2.

No 563 Eva L Ragnemalm: Student Modelling based on Collaborative Dialogue with a Learning Companion, 1999, ISBN 91-7219-412-X.

No 567 Jörgen Lindström: Does Distance matter? On geographical dispersion in organisations, 1999, ISBN 917219-439-1.

No 582 Vanja Josifovski: Design, Implementation and Evaluation of a Distributed Mediator System for Data Integration, 1999, ISBN 91-7219-482-0.

No 589 Rita Kovordányi: Modeling and Simulating Inhibitory Mechanisms in Mental Image Reinterpretation - Towards Cooperative HumanComputer Creativity, 1999, ISBN 91-7219-506-1.

No 592 Mikael Ericsson: Supporting the Use of Design Knowledge - An Assessment of Commenting Agents, 1999, ISBN 91-7219-532-0.

No 593 Lars Karlsson: Actions, Interactions and Narratives, 1999, ISBN 91-7219-534-7.

No 594 C. G. Mikael Johansson: Social and Organizational Aspects of Requirements Engineering Methods - A practice-oriented approach, 1999, ISBN 91-7219-541$\mathrm{X}$.

No 595 Jörgen Hansson: Value-Driven Multi-Class Overload Management in Real-Time Database Systems, 1999, ISBN 91-7219-542-8.

No 596 Niklas Hallberg: Incorporating User Values in the Design of Information Systems and Services in the Public Sector: A Methods Approach, 1999, ISBN 917219-543-6.

No 597 Vivian Vimarlund: An Economic Perspective on the Analysis of Impacts of Information Technology: From Case Studies in Health-Care towards General Models and Theories, 1999, ISBN 91-7219-544-4.

No 598 Johan Jenvald: Methods and Tools in ComputerSupported Taskforce Training, 1999, ISBN 91-7219547-9.

No 607 Magnus Merkel: Understanding and enhancing translation by parallel text processing, 1999, ISBN 917219-614-9.

No 611 Silvia Coradeschi: Anchoring symbols to sensory data, 1999, ISBN 91-7219-623-8.

No 613 Man Lin: Analysis and Synthesis of Reactive Systems: A Generic Layered Architecture Perspective, 1999, ISBN 91-7219-630-0.

No 618 Jimmy Tjäder: Systemimplementering i praktiken En studie av logiker i fyra projekt, 1999, ISBN 917219-657-2.

No 627 Vadim Engelson: Tools for Design, Interactive Simulation, and Visualization of Object-Oriented Models in Scientific Computing, 2000, ISBN 91-7219709-9. 
No 637 Esa Falkenroth: Database Technology for Control and Simulation, 2000, ISBN 91-7219-766-8.

No 639 Per-Arne Persson: Bringing Power and Knowledge Together: Information Systems Design for Autonomy and Control in Command Work, 2000, ISBN 91-7219796-X.

No 660 Erik Larsson: An Integrated System-Level Design for Testability Methodology, 2000, ISBN 91-7219-890-7.

No 688 Marcus Bjäreland: Model-based Execution Monitoring, 2001, ISBN 91-7373-016-5.

No 689 Joakim Gustafsson: Extending Temporal Action Logic, 2001, ISBN 91-7373-017-3.

No 720 Carl-Johan Petri: Organizational Information Provision - Managing Mandatory and Discretionary Use of Information Technology, 2001, ISBN-91-7373-1269.

No 724 Paul Scerri: Designing Agents for Systems with Adjustable Autonomy, 2001, ISBN 9173732079.

No 725 Tim Heyer: Semantic Inspection of Software Artifacts: From Theory to Practice, 2001, ISBN 91 73732087.

No 726 Pär Carlshamre: A Usability Perspective on Requirements Engineering - From Methodology to Product Development, 2001, ISBN 9173732125.

No 732 Juha Takkinen: From Information Management to Task Management in Electronic Mail, 2002, ISBN 91 73732583.

No 745 Johan Åberg: Live Help Systems: An Approach to Intelligent Help for Web Information Systems, 2002, ISBN 91-7373-311-3.

No 746 Rego Granlund: Monitoring Distributed Teamwork Training, 2002, ISBN 91-7373-312-1.

No 757 Henrik André-Jönsson: Indexing Strategies for Time Series Data, 2002, ISBN 917373-346-6.

No 747 Anneli Hagdahl: Development of IT-supported Interorganisational Collaboration - A Case Study in the Swedish Public Sector, 2002, ISBN 91-7373-314-8.

No 749 Sofie Pilemalm: Information Technology for NonProfit Organisations - Extended Participatory Design of an Information System for Trade Union Shop Stew ard s, 2002, ISBN 91-7373-318-0.

No 765 Stefan Holmlid: Adapting users: Towards a theory of use quality, 2002, ISBN 91-7373-397-0.

No 771 Magnus Morin: Multimedia Representations of Distributed Tactical Operations, 2002, ISBN 91-7373-4217.

No 772 Pawel Pietrzak: A Type-Based Framework for Locating Errors in Constraint Logic Programs, 2002, ISBN 91-7373-422-5.

No 758 Erik Berglund: Library Communication Among Programmers Worldwide, 2002, ISBN 91-7373-349-0.

No 774 Choong-ho Yi: Modelling Object-Oriented Dynamic Systems Using a Logic-Based Framew ork, 2002, ISBN 91-7373-424-1.

No 779 Mathias Broxvall: A Study in the Computational Complexity of Temporal Reasoning, 2002, ISBN 917373-440-3

No 793 Asmus Pandikow: A Generic Principle for Enabling Interoperability of Structured and Object-Oriented Analysis and Design Tools, 2002, ISBN 91-7373-479-9.

No 785 Lars Hult: Publika Informationstjänster. En studie av den Internetbaserade encyklopedins bruksegenskaper, 2003, ISBN 91-7373-461-6.

No 800 Lars Taxén: A Framework for the Coordination of Complex Systems' Development, 2003, ISBN 917373-604-X
No 808 Klas Gäre: Tre perspektiv på förväntningar och förändringar i samband med införande av informationssystem, 2003, ISBN 91-7373-618-X.

No 821 Mikael Kindborg: Concurrent Comics programming of social agents by children, 2003, ISBN 91-7373-651-1.

No 823 Christina Ölvingson: On Development of Information Systems with GIS Functionality in Public Health Informatics: A Requirements Engineering Approach, 2003, ISBN 91-7373-656-2.

No 828 Tobias Ritzau: Memory Efficient Hard Real-Time Garbage Collection, 2003, ISBN 91-7373-666-X.

No 833 Paul Pop: Analysis and Synthesis of Communication-Intensive Heterogeneous Real-Time Systems, 2003, ISBN 91-7373-683-X.

No 852 Johan Moe: Observing the Dynamic Behaviour of Large Distributed Systems to Improve Development and Testing - An Empirical Study in Software Engineering, 2003, ISBN 91-7373-779-8.

No 867 Erik Herzog: An Approach to Systems Engineering Tool Data Representation and Exchange, 2004, ISBN 91-7373-929-4.

No 872 Aseel Berglund: Augmenting the Remote Control: Studies in Complex Information Navigation for Digital TV, 2004, ISBN 91-7373-940-5.

No 869 Jo Skåmedal: Telecommuting's Implications on Travel and Travel Patterns, 2004, ISBN 91-7373-935-9.

No 870 Linda Askenäs: The Roles of IT - Studies of Organising when Implementing and Using Enterprise Systems, 2004, ISBN 91-7373-936-7.

No 874 Annika Flycht-Eriksson: Design and Use of Ontologies in Information-Providing Dialogue Systems, 2004, ISBN 91-7373-947-2.

No 873 Peter Bunus: Debugging Techniques for EquationBased Languages, 2004, ISBN 91-7373-941-3.

No 876 Jonas Mellin: Resource-Predictable and Efficient Monitoring of Events, 2004, ISBN 91-7373-956-1.

No 883 Magnus Bång: Computing at the Speed of Paper: Ubiquitous Computing Environments for Healthcare Professionals, 2004, ISBN 91-7373-971-5

No 882 Robert Eklund: Disfluency in Swedish humanhuman and human-machine travel booking dialogues, 2004, ISBN 91-7373-966-9.

No 887 Anders Lindström: English and other Foreign Linguistic Elements in Spoken Swedish. Studies of Productive Processes and their Modelling using Finite-State Tools, 2004, ISBN 91-7373-981-2

No 889 Zhiping Wang: Capacity-Constrained Production-inventory systems - Modelling and Analysis in both a traditional and an e-business context, 2004, ISBN 9185295-08-6.

No 893 Pernilla Qvarfordt: Eyes on Multimodal Interaction, 2004, ISBN 91-85295-30-2.

No 910 Magnus Kald: In the Borderland between Strategy and Management Control - Theoretical Framework and Empirical Evidence, 2004, ISBN 91-85295-82-5.

No 918 Jonas Lundberg: Shaping Electronic News: Genre Perspectives on Interaction Design, 2004, ISBN 9185297-14-3.

No 900 Mattias Arvola: Shades of use: The dynamics of interaction design for sociable use, 2004, ISBN 91$85295-42-6$

No 920 Luis Alejandro Cortés: Verification and Scheduling Techniques for Real-Time Embedded Systems, 2004, ISBN 91-85297-21-6.

No 929 Diana Szentivanyi: Performance Studies of FaultTolerant Middlew are, 2005, ISBN 91-85297-58-5. 
No 933 Mikael Cäker: Management Accounting as Constructing and Opposing Customer Focus: Three Case Studies on Management Accounting and Customer Relations, 2005, ISBN 91-85297-64-X.

No 937 Jonas Kvarnström: TALplanner and Other Extensions to Temporal Action Logic, 2005, ISBN 9185297-75-5.

No 938 Bourhane Kadmiry: Fuzzy Gain-Scheduled Visual Servoing for Unmanned Helicopter, 2005, ISBN 9185297-76-3.

No 945 Gert Jervan: Hybrid Built-In Self-Test and Test Generation Techniques for Digital Systems, 2005, ISBN : 91-85297-97-6.

No 946 Anders Arpteg: Intelligent Semi-Structured Information Extraction, 2005, ISBN 91-85297-98-4.

No 947 Ola Angelsmark: Constructing Algorithms for Constraint Satisfaction and Related Problems - Methods and Applications, 2005, ISBN 91-85297-99-2.

No 963 Calin Curescu: Utility-based Optimisation of Resource Allocation for Wireless Networks, 2005, ISBN 91-85457-07-8.

No 972 Björn Johansson: Joint Control in Dynamic Situations, 2005, ISBN 91-85457-31-0.

No 974 Dan Lawesson: An Approach to Diagnosability Analysis for Interacting Finite State Systems, 2005, ISBN 91-85457-39-6.

No 979 Claudiu Duma: Security and Trust Mechanisms for Groups in Distributed Services, 2005, ISBN 91-8545754-X.

No 983 Sorin Manolache: Analysis and Optimisation of Real-Time Systems with Stochastic Behaviour, 2005, ISBN 91-85457-60-4.

No 986 Yuxiao Zhao: Standards-Based Application Integration for Business-to-Business Communications, 2005, ISBN 91-85457-66-3.

No 1004 Patrik Haslum: Admissible Heuristics for Automated Planning, 2006, ISBN 91-85497-28-2.

No 1005 Aleksandra Tešanovic: Developing Reusable and Reconfigurable Real-Time Software using Aspects and Components, 2006, ISBN 91-85497-29-0.

No 1008 David Dinka: Role, Identity and Work: Extending the design and development agenda, 2006, ISBN 9185497-42-8.

No 1009 Iakov Nakhimovski: Contributions to the Modeling and Simulation of Mechanical Systems with Detailed Contact Analysis, 2006, ISBN 91-85497-43-X.

No 1013 Wilhelm Dahllöf: Exact Algorithms for Exact Satisfiability Problems, 2006, ISBN 91-85523-97-6.

No 1016 Levon Saldamli: PDEModelica - A High-Level Language for Modeling with Partial Differential Equations, 2006, ISBN 91-85523-84-4.

No 1017 Daniel Karlsson: Verification of Component-based Embedded System Designs, 2006, ISBN 91-85523-79-8

No 1018 Ioan Chisalita: Communication and Networking Techniques for Traffic Safety Systems, 2006, ISBN 9185523-77-1.

No 1019 Tarja Susi: The Puzzle of Social Activity - The Significance of Tools in Cognition and Cooperation, 2006, ISBN 91-85523-71-2.

No 1021 Andrzej Bednarski: Integrated Optimal Code Generation for Digital Signal Processors, 2006, ISBN 9185523-69-0.

No 1022 Peter Aronsson: Automatic Parallelization of Equation-Based Simulation Programs, 2006, ISBN 9185523-68-2.
No 1030 Robert Nilsson: A Mutation-based Framework for Automated Testing of Timeliness, 2006, ISBN 9185523-35-6.

No 1034 Jon Edvardsson: Techniques for Automatic Generation of Tests from Programs and Specifications, 2006, ISBN 91-85523-31-3.

No 1035 Vaida Jakoniene: Integration of Biological Data, 2006, ISBN 91-85523-28-3.

No 1045 Genevieve Gorrell: Generalized Hebbian Algorithms for Dimensionality Reduction in Natural Language Processing, 2006, ISBN 91-85643-88-2.

No 1051 Yu-Hsing Huang: Having a New Pair of Glasses Applying Systemic Accident Models on Road Safety, 2006, ISBN 91-85643-64-5.

No 1054 Åsa Hedenskog: Perceive those things which cannot be seen - A Cognitive Systems Engineering perspective on requirements management, 2006, ISBN 91-85643-57-2.

No 1061 Cécile Åberg: An Evaluation Platform for Semantic Web Technology, 2007, ISBN 91-85643-31-9.

No 1073 Mats Grindal: Handling Combinatorial Explosion in Software Testing, 2007, ISBN 978-91-85715-74-9.

No 1075 Almut Herzog: Usable Security Policies for Runtime Environments, 2007, ISBN 978-91-85715-65-7.

No 1079 Magnus Wahlström: Algorithms, measures, and upper bounds for Satisfiability and related problems, 2007, ISBN 978-91-85715-55-8.

No 1083 Jesper Andersson: Dynamic Software Architectures, 2007, ISBN 978-91-85715-46-6.

No 1086 Ulf Johansson: Obtaining Accurate and Comprehensible Data Mining Models - An Evolutionary Approach, 2007, ISBN 978-91-85715-34-3.

No 1089 Traian Pop: Analysis and Optimisation of Distributed Embedded Systems with Heterogeneous Scheduling Policies, 2007, ISBN 978-91-85715-27-5.

No 1091 Gustav Nordh: Complexity Dichotomies for CSPrelated Problems, 2007, ISBN 978-91-85715-20-6.

No 1106 Per Ola Kristensson: Discrete and Continuous Shape Writing for Text Entry and Control, 2007, ISBN 97891-85831-77-7.

No 1110 He Tan: Aligning Biomedical Ontologies, 2007, ISBN 978-91-85831-56-2.

No 1112 Jessica Lindblom: Minding the body - Interacting socially through embodied action, 2007, ISBN 978-9185831-48-7.

No 1113 Pontus Wärnestål: Dialogue Behavior Management in Conversational Recommender Systems, 2007, ISBN 978-91-85831-47-0.

No 1120 Thomas Gustafsson: Management of Real-Time Data Consistency and Transient Overloads in Embedded Systems, 2007, ISBN 978-91-85831-33-3.

No 1127 Alexandru Andrei: Energy Efficient and Predictable Design of Real-time Embedded Systems, 2007, ISBN 978-91-85831-06-7.

No 1139 Per Wikberg: Eliciting Knowledge from Experts in Modeling of Complex Systems: Managing Variation and Interactions, 2007, ISBN 978-91-85895-66-3.

No 1143 Mehdi Amirijoo: QoS Control of Real-Time Data Services under Uncertain Workload, 2007, ISBN 97891-85895-49-6.

No 1150 Sanny Syberfeldt: Optimistic Replication with Forward Conflict Resolution in Distributed Real-Time Databases, 2007, ISBN 978-91-85895-27-4.

No 1155 Beatrice Alenljung: Envisioning a Future Decision Support System for Requirements Engineering - A Holistic and Human-centred Perspective, 2008, ISBN 978-91-85895-11-3. 
No 1156 Artur Wilk: Types for XML with Application to Xcerpt, 2008, ISBN 978-91-85895-08-3.

No 1183 Adrian Pop: Integrated Model-Driven Development Environments for Equation-Based Object-Oriented Languages, 2008, ISBN 978-91-7393-895-2.

No 1185 Jörgen Skågeby: Gifting Technologies Ethnographic Studies of End-users and Social Media Sharing, 2008, ISBN 978-91-7393-892-1.

No 1187 Imad-Eldin Ali Abugessaisa: Analytical tools and information-sharing methods supporting road safety organizations, 2008, ISBN 978-91-7393-887-7.

No 1204 H. Joe Steinhauer: A Representation Scheme for Description and Reconstruction of Object Configurations Based on Qualitative Relations, 2008, ISBN 978-91-7393-823-5.

No 1222 Anders Larsson: Test Optimization for Core-based System-on-Chip, 2008, ISBN 978-91-7393-768-9.

No 1238 Andreas Borg: Processes and Models for Capacity Requirements in Telecommunication Systems, 2009, ISBN 978-91-7393-700-9.

No 1240 Fredrik Heintz: DyKnow: A Stream-Based Knowledge Processing Middleware Framework, 2009, ISBN 978-91-7393-696-5.

No 1241 Birgitta Lindström: Testability of Dynamic RealTime Systems, 2009, ISBN 978-91-7393-695-8.

No 1244 Eva Blomqvist: Semi-automatic Ontology Construction based on Patterns, 2009, ISBN 978-91-7393-683-5.

No 1249 Rogier Woltjer: Functional Modeling of Constraint Management in Aviation Safety and Command and Control, 2009, ISBN 978-91-7393-659-0.

No 1260 Gianpaolo Conte: Vision-Based Localization and Guidance for Unmanned Aerial Vehicles, 2009, ISBN 978-91-7393-603-3.

No 1262 AnnMarie Ericsson: Enabling Tool Support for Formal Analysis of ECA Rules, 2009, ISBN 978-91-7393598-2.

No 1266 Jiri Trnka: Exploring Tactical Command and Control: A Role-Playing Simulation Approach, 2009, ISBN 978-91-7393-571-5.

No 1268 Bahlol Rahimi: Supporting Collaborative Work through ICT - How End-users Think of and Adopt Integrated Health Information Systems, 2009, ISBN 978-91-7393-550-0.

No 1274 Fredrik Kuivinen: Algorithms and Hardness Results for Some Valued CSPs, 2009, ISBN 978-91-7393-525-8.

No 1281 Gunnar Mathiason: Virtual Full Replication for Scalable Distributed Real-Time Databases, 2009, ISBN 978-91-7393-503-6.

No 1290 Viacheslav Izosimov: Scheduling and Optimization of Fault-Tolerant Distributed Embedded Systems, 2009, ISBN 978-91-7393-482-4.

No 1294 Johan Thapper: Aspects of a Constraint Optimisation Problem, 2010, ISBN 978-91-7393-464-0.

No 1306 Susanna Nilsson: Augmentation in the Wild: User Centered Development and Evaluation of Augmented Reality Applications, 2010, ISBN 978-917393-416-9.

No 1313 Christer Thörn: On the Quality of Feature Models, 2010, ISBN 978-91-7393-394-0.

No 1321 Zhiyuan He: Temperature Aware and DefectProbability Driven Test Scheduling for System-onChip, 2010, ISBN 978-91-7393-378-0.

No 1333 David Broman: Meta-Languages and Semantics for Equation-Based Modeling and Simulation, 2010, ISBN 978-91-7393-335-3.

No 1337 Alexander Siemers: Contributions to Modelling and Visualisation of Multibody Systems Simulations with
Detailed Contact Analysis, 2010, ISBN 978-91-7393317-9.

No 1354 Mikael Asplund: Disconnected Discoveries: Availability Studies in Partitioned Networks, 2010, ISBN 978-91-7393-278-3.

No 1359 Jana Rambusch: Mind Games Extended: Understanding Gameplay as Situated Activity, 2010, ISBN 978-91-7393-252-3.

No 1373 Sonia Sangari: Head Movement Correlates to Focus Assignment in Swedish,2011,ISBN 978-91-7393-154-0.

No 1374 Jan-Erik Källhammer: Using False Alarms when Developing Automotive Active Safety Systems, 2011, ISBN 978-91-7393-153-3.

No 1375 Mattias Eriksson: Integrated Code Generation, 2011, ISBN 978-91-7393-147-2.

No 1381 Ola Leifler: Affordances and Constraints of Intelligent Decision Support for Military Command and Control - Three Case Studies of Support Systems, 2011, ISBN 978-91-7393-133-5.

No 1386 Soheil Samii: Quality-Driven Synthesis and Optimization of Embedded Control Systems, 2011, ISBN 978-91-7393-102-1.

No 1419 Erik Kuiper: Geographic Routing in Intermittentlyconnected Mobile Ad Hoc Networks: Algorithms and Performance Models, 2012, ISBN 978-91-7519981-8.

No 1451 Sara Stymne: Text Harmonization Strategies for Phrase-Based Statistical Machine Translation, 2012, ISBN 978-91-7519-887-3.

No 1455 Alberto Montebelli: Modeling the Role of Energy Management in Embodied Cognition, 2012, ISBN 978-91-7519-882-8

No 1465 Mohammad Saifullah: Biologically-Based Interactive Neural Network Models for Visual Attention and Object Recognition, 2012, ISBN 978-91-7519-838-5.

No 1490 Tomas Bengtsson: Testing and Logic Optimization Techniques for Systems on Chip, 2012, ISBN 978-917519-742-5.

No 1481 David Byers: Improving Software Security by Preventing Known Vulnerabilities, 2012, ISBN 97891-7519-784-5.

No 1496 Tommy Färnqvist: Exploiting Structure in CSPrelated Problems, 2013, ISBN 978-91-7519-711-1.

No 1503 John Wilander: Contributions to Specification, Implementation, and Execution of Secure Software, 2013, ISBN 978-91-7519-681-7.

No 1506 Magnus Ingmarsson: Creating and Enabling the Useful Service Discovery Experience, 2013, ISBN 97891-7519-662-6.

No 1547 Wladimir Schamai: Model-Based Verification of Dynamic System Behavior against Requirements: Method, Language, and Tool, 2013, ISBN 978-917519-505-6.

No 1551 Henrik Svensson: Simulations, 2013, ISBN 978-917519-491-2.

No 1559 Sergiu Rafiliu: Stability of Adaptive Distributed Real-Time Systems with Dynamic Resource Management, 2013, ISBN 978-91-7519-471-4.

No 1581 Usman Dastgeer: Performance-aware Component Composition for GPU-based Systems, 2014, ISBN 978-91-7519-383-0

No 1602 Cai Li: Reinforcement Learning of Locomotion based on Central Pattern Generators, 2014, ISBN 978-917519-313-7.

No 1652 Roland Samlaus: An Integrated Development Environment with Enhanced Domain-Specific 
Interactive Model Validation, 2015, ISBN 978-917519-090-7.

No 1663 Hannes Uppman: On Some Combinatorial Optimization Problems: Algorithms and Complexity, 2015, ISBN 978-91-7519-072-3.

No 1664 Martin Sjölund: Tools and Methods for Analysis, Debugging, and Performance Improvement of Equation-Based Models, 2015, ISBN 978-91-7519-071-6.

No 1666 Kristian Stavåker: Contributions to Simulation of Modelica Models on Data-Parallel Multi-Core Architectures, 2015, ISBN 978-91-7519-068-6.

No 1680 Adrian Lifa: Hardware/ Software Codesign of Embedded Systems with Reconfigurable and Heterogeneous Platforms, 2015, ISBN 978-91-7519-0402.

No 1685 Bogdan Tanasa: Timing Analysis of Distributed Embedded Systems with Stochastic Workload and Reliability Constraints, 2015, ISBN 978-91-7519-022-8.

No 1691 Håkan Warnquist: Troubleshooting Trucks Automated Planning and Diagnosis, 2015, ISBN 97891-7685-993-3.

No 1702 Nima Aghaee: Thermal Issues in Testing of Advanced Systems on Chip, 2015, ISBN 978-91-7685949-0.

No 1715 Maria Vasilevskaya: Security in Embedded Systems: A Model-Based Approach with Risk Metrics, 2015, ISBN 978-91-7685-917-9.

No 1729 Ke Jiang: Security-Driven Design of Real-Time Embedded System, 2016, ISBN 978-91-7685-884-4.

No 1733 Victor Lagerkvist: Strong Partial Clones and the Complexity of Constraint Satisfaction Problems: Limitations and Applications, 2016, ISBN 978-91-7685856-1.

No 1734 Chandan Roy: An Informed System Development Approach to Tropical Cyclone Track and Intensity Forecasting, 2016, ISBN 978-91-7685-854-7.

No 1746 Amir Aminifar: Analysis, Design, and Optimization of Embedded Control Systems, 2016, ISBN 978-917685-826-4.

No 1747 Ekhiotz Vergara: Energy Modelling and Fairness for Efficient Mobile Communication, 2016, ISBN 978-91-7685-822-6.

No 1748 Dag Sonntag: Chain Graphs - Interpretations, Expressiveness and Learning Algorithms, 2016, ISBN 978-91-7685-818-9.

No 1768 Anna Vapen: Web Authentication using ThirdParties in Untrusted Environments, 2016, ISBN 978-91-7685-753-3.

No 1778 Magnus Jandinger: On a Need to Know Basis: A Conceptual and Methodological Framework for Modelling and Analysis of Information Demand in an Enterprise Context, 2016, ISBN 978-91-7685-713-7.

No 1798 Rahul Hiran: Collaborative Network Security: Targeting Wide-area Routing and Edgenetw ork Attacks, 2016, ISBN 978-91-7685-662-8.

No 1813 Nicolas Melot: Algorithms and Framework for Energy Efficient Parallel Stream Computing on Many-Core Architectures, 2016, ISBN 978-91-7685623-9.

No 1823 Amy Rankin: Making Sense of Adaptations: Resilience in High-Risk Work, 2017, ISBN 978-917685-596-6.
No 1831 Lisa Malmberg: Building Design Capability in the Public Sector: Expanding the Horizons of Development, 2017, ISBN 978-91-7685-585-0.

No 1851 Marcus Bendtsen: Gated Bayesian Networks, 2017, ISBN 978-91-7685-525-6.

No 1852 Zlatan Dragisic: Completion of Ontologies and Ontology Networks, 2017, ISBN 978-91-7685-522-5.

No 1854 Meysam Aghighi: Computational Complexity of some Optimization Problems in Planning, 2017, ISBN 978-91-7685-519-5.

Linköping Studies in Arts and Science

No 504 Ing-Marie Jonsson: Social and Emotional Characteristics of Speech-based In-Vehicle Information Systems: Impact on Attitude and Driving Behaviour, 2009, ISBN 978-91-7393-478-7.

No 586 Fabian Segelström: Stakeholder Engagement for Service Design: How service designers identify and communicate insights, 2013, ISBN 978-91-7519-554-4.

No 618 Johan Blomkvist: Representing Future Situations of Service: Prototyping in Service Design, 2014, ISBN 978-91-7519-343-4.

No 620 Marcus Mast: Human-Robot Interaction for SemiAutonomous Assistive Robots, 2014, ISBN 978-917519-319-9.

No 677 Peter Berggren: Assessing Shared Strategic Understanding, 2016, ISBN 978-91-7685-786-1.

No 695 Mattias Forsblad: Distributed cognition in home environments: The prospective memory and cognitive practices of older adults, 2016, ISBN 97891-7685-686-4.

\section{Linköping Studies in Statistics}

No 9 Davood Shahsavani: Computer Experiments Designed to Explore and Approximate Complex Deterministic Models, 2008, ISBN 978-91-7393-976-8.

No 10 Karl Wahlin: Roadmap for Trend Detection and Assessment of Data Quality, 2008, ISBN 978-91-7393792-4.

No 11 Oleg Sysoev: Monotonic regression for large multivariate datasets, 2010, ISBN 978-91-7393-412-1.

No 13 Agné Burauskaite-Harju: Characterizing Temporal Change and Inter-Site Correlations in Daily and Subdaily Precipitation Extremes, 2011, ISBN 978-91-7393110-6.

\section{Linköping Studies in Information Science}

No 1 Karin Axelsson: Metodisk systemstrukturering- att skapa samstämmighet mellan informationssystemarkitektur och verksamhet, 1998. ISBN-9172-19-296-8.

No 2 Stefan Cronholm: Metodverktyg och användbarhet en studie av datorstödd metodbaserad systemutveckling, 1998, ISBN-9172-19-299-2.

No 3 Anders Avdic: Användare och utvecklare - om anveckling med kalkylprogram, 1999. ISBN-91-7219606-8.

No 4 Owen Eriksson: Kommunikationskvalitet hos informationssystem och affärsprocesser, 2000, ISBN 917219-811-7.

No 5 Mikael Lind: Från system till process - kriterier för processbestämning vid verksamhetsanalys, 2001, ISBN 91-7373-067-X.

No 6 Ulf Melin: Koordination och informationssystem i företag och nätverk, 2002, ISBN 91-7373-278-8. 
No 7 Pär J. Ågerfalk: Information Systems Actability - Understanding Information Technology as a Tool for Business Action and Communication, 2003, ISBN 917373-628-7.

No 8 Ulf Seigerroth: Att förstå och förändra systemutvecklingsverksamheter - en taxonomi för metautveckling, 2003, ISBN91-7373-736-4.

No 9 Karin Hedström: Spår av datoriseringens värden Effekter av IT i äldreomsorg, 2004, ISBN 91-7373-9634.

No 10 Ewa Braf: Knowledge Demanded for Action Studies on Knowledge Mediation in Organisations, 2004, ISBN 91-85295-47-7.

No 11 Fredrik Karlsson: Method Configuration method and computerized tool support, 2005, ISBN 91-8529748-8.

No 12 Malin Nordström: Styrbar systemförvaltning - Att organisera system förvaltningsverksamhet med hjälp av effektiva förvaltningsobjekt, 2005, ISBN 91-8529760-7.

No 13 Stefan Holgersson: Yrke: POLIS - Yrkeskunskap, motivation, IT-system och andra förutsättningar för polisarbete, 2005, ISBN 91-85299-43-X.

No 14 Benneth Christiansson, Marie-Therese Christiansson: Mötet mellan process och komponent - mot ett ramverk för en verksamhetsnära kravspecifikation vid anskaffning av komponentbaserade informationssystem, 2006, ISBN 91-85643$22-\mathrm{X}$. 